\title{
Methods and Analysis of Collar Strategies
}

\author{
N. El-Hassan \\ University of Technology, Sydney
}

\author{
A. Hall \\ NCER, Queensland University of Technology
}

\author{
I. Tulunay \\ University of New South Wales
}

\begin{abstract}
An equity portfolio with a collar strategy consists of a long position in the underlying index together with long put options for insurance and short call options to mitigate the cost of insurance or enhance the return. Using the S\&P/ASX 200 index, this study investigates the performances of fourteen collar strategies and for comparison two protective put strategies. We consider two simulations: one with traded option market data and the other utilizing Black-Scholes option prices. The investigation period is 2008-2016 and we consider three sub-periods representing different market conditions. The active collar strategies considered follow those introduced by Szado \& Schneeweis (2010a). As a summary of our results, the best performing strategies are a protective put and a zero-cost collar.
\end{abstract}

Keywords: option strategies, collar strategy, protective puts, covered-call, buy-write strategy, equity index portfolio with option strategies, portfolio performance, portfolio management

\section{INTRODUCTION}

A collar strategy is a combination of covered call (buy-write) and protective put strategies. The covered call strategy involves writing call options against a long position in an underlying asset. Empirical evidence indicates that covered call strategies, in general, produce better risk-adjusted returns than holding naked long positions in the underlying assets (see for example, El-Hassan et al. (2017)). Protective puts offer protection for a portfolio for a pre-specified lower bound. Collar strategies reduce the total risk and loss risk (lower semi-deviation) (Dublin (2010)) of portfolios. This study also shows that all examined collar and protective put strategies produce better risk-adjusted returns than pure stock strategies.

We emphasize that the use of mean-variance analysis is not an appropriate framework for evaluating the performance of the portfolios with option strategies as the returns are not normally distributed (see Bookstaber \& Clarke (1985) and Leggio \& Lien (2008)). Therefore, traditional risk-adjusted measures used for performance analysis, such as Sharpe ratio, can be misleading. Our performance analysis relies on the Adjusted Sharpe ratio, the Excess Return- Entropy ratio and the Stutzer Index (formulae given in Appendix). The Adjusted Sharpe ratio accounts for skewness and kurtosis. The Excess Return-Entropy ratio is obtained by replacing the standard deviation by Shannon entropy in the Sharpe ratio. The entropy 
accounts for higher moments of a distribution and offers a better explanation of the distribution (see Ebrahimi et al. (1999), Milev et al. (2012)). The advantages of the entropy measure over the standard deviation were discussed by Philippatos \& Wilson (1972) who compared the mean-entropy approach with the traditional mean-variance approach in portfolio theory. Usta \& Kantar (2011) introduced meanvariance-skewness-entropy model as multi-objective portfolio optimization and found that this model performed better than the traditional portfolio selection models in out-of-sample tests. Ormos \& Zibriczky (2014) defined continuous entropy as an alternative measure of risk for asset pricing. Their regression results show that entropy has higher explanatory power for the expected return than the capital asset pricing model (CAPM) beta and the standard deviation. Lassance \& Vrins (2017) proposed the exponential R'enyi entropy as an objective function for portfolio selection under non-normality. Based on these results, we included excess return- entropy measure in our performance analysis. The Stutzer index is the rate at which the probability of under-performance approaches zero. The highest the Stutzer index indicates the best investment. ${ }^{1}$

CBOE (2012) documented that "Equity collars are used by investors whose primary concern is the downside risk of a stock position. They are willing to place a cap on upside potential to limit their downside risk at little, and sometimes no cost. Collars may be of special interest to those investors who have one equity position that accounts for a large proportion of their net worth, and who may not be able to reduce the size of this position. For these investors, low-cost protection may take a precedence over maintaining upside potential." One reason for popularity of collar strategies is the mitigation of the cost of insurance (buying protective put) by writing call options. However, we need to emphasize the high risk associated with writing call options which could be exercised (see Appendix for the percentages of call exercise cost over the holding period to the initial investment for all collar portfolios). Unexpected sharp increase in the index would result in a substantial loss of investment. For example, after the Global Financial Criss (GFC) in 2008-2009, the index returns from 4-Sep-2009 to 1-Oct-2009 is 6\% (70\% annualized).

In this study, we analyze seventeen different strategies under different market conditions, including the extreme market movements (GFC period). Investigating the performance of the strategies in the Australian market during and after GFC is particularly interesting. We chose three subperiods with intervals of four years (two years overlapping). Without overlapping periods, we would have subperiods of two years which would consist of too few quarters. By allowing overlapping periods, working on subperiods of four years is justifiable. The first subperiod 2008-2012 includes the Global Financial Criss (GFC) event. The second subperiod 2010-2014 contains negative quarterly returns 59\% of time and it represents bear and then bull market conditions. The last subperiod 2012-2016 contains positive quarterly returns $94 \%$ of time, representing bull market conditions.

Abid et al. (2007) examine the performances of unhedged to hedged positions for ten main stocks and indices with writing OTM (Out-Of-Money) call options and buying ITM (In-The-Money) put options in the French market for one year during 1999. Their tests showed that both hedged positions, writing OTM covered call and buying ITM protective put, are superior to their corresponding naked stock strategy. In our study, we use OTM options for both calls and puts. Buying put options plays the role of insurance for the portfolios and creates a lower bound below the spot index level whereas an ITM put option creates a higher lower bound than OTM put option.

In understanding the performance of collar strategies, Figelman (2008) decompose the expected return of a covered call strategy in terms of risk-free return, equity risk premium and call risk premium. Israelov \& Nielsen (2015) consider the covered call-in terms of the exposures of passive equity, short volatility and dynamic equity. Using the decomposition of Israelov \& Nielsen (2015), Israelov \& Klein (2016) examined the performances of CBOE S\&P 500 95-110 Collar Index from July 1986 to December 2014. CBOE S\&P 500 95-110 Collar index consists of a long position in the S\&P 500, a long 3 months 5\% OTM put option and short monthly $10 \%$ OTM call option. In contrast to the usual perception that the collar should have the same return as its underlying security, they argue that a typical collar return should be expected to be lower than its underlying because (1) it has lower equity exposure, and (2) it purchases put options that are more expensive than the call options to sell and thus pays volatility risk premium. They suggest selling a portion of the equity portfolio instead, if one wishes to reduce the equity risk in a simple, elegant, effective, and 
transparent way. Israelov et al. (2017) decompose the S\&P 500 index returns into the downside (covered call) and upside (long call) exposures. They conclude that it is better to reduce the allocation to the asset class rather than to buy insurance through options if the downside risk in a given asset class is too much to bear.

Szado \& Kazemi (2009) examined risk-return characteristic of a passive long collar strategy on the QQQ ETF from Mar-1999 to Mar-2008 using 27 variations on the strategy by altering the time to expiration and the moneyness OTM and ATM (At-The-Money) for both call and put. They found that 6 months put, 1 month call collar provides far superior returns to the QQQ buy and hold strategy at about a third of the volatility. Their analysis confirms that the effectiveness of the collar strategy as a protective strategy. They also observed that most of the collar implementations exhibit a positive Stutzer index, in contrast to the negative Stutzer index of the QQQ. Our study shows similar results in the following sense: the Stutzer index of the naked stock strategy is the minimum among all portfolios with collar and protective put strategies for all periods that we examined.

Szado \& Schneeweis (2010a) uses QQQ ETF index options from Chicago Board Options Exchange (CBOE) Market over the period March 1999 to May 2009 (up- dated data version March 1999 to September 2010 Szado \& Schneeweis (2010b)). The CBOE Volatility Index (VIX Index) is based on S\&P 500. Szado \& Schneeweis (2010a) introduced an active collar strategy based on volatility, momentum, and macroeconomic signals. We explain their methods in detail in Section 3.1. We ap ply their active strategies to six different scenarios and make use of S\&P/ASX 200 Volatility Index, AXVIVOL (introduced by ASX in 2008). Furthermore, we propose a new active collar strategy in which we also use AXVIVOL.

In the light of their work, we implement our strategies with semi-annually balancing put options to reduce the cost of protection and quarterly balancing OTM call options with moneyness range from $2 \%$ to $7 \%$. Writing $10 \%$ OTM call options would not produce significant positive cash flow. However, high OTM percentage reduces the risk of the exercise cost for the call sold. As Australian option market is illiquid, we balance our portfolios quarterly; call options are written quarterly and protective put options are bought every six months. Buying put options quarterly would have been an expensive protection strategy.

\section{DATA}

The data used in this study, the sources and codes are described in Table 1. The ASX volatility index reflects expected equity market volatility over the next 30 days utilizing the mid prices for S\&P/ASX 200 put and call options to calculate a weighted average of the implied volatility of the options (see DJI (2017) for more on VIX methodology). We use the Bank Accepted Bills/Negotiable Certificates of Deposit 1 month (1-month BABs/NCDs) from the Reserve Bank of Australia (RBA) as an approximate risk-free rate. The business cycles for the period 2004-2014, obtained from FRED (Federal Reserve Bank of St. Louis), are derived from the OECD based Recession Indicators for Australia following the Peak through the Trough methodology. We calculate the business cycles for the period $2004-20162$.

TABLE 1

DATA AND DATA SOURCES

\begin{tabular}{|l|l|l|}
\hline Data & Data Source & Code \\
\hline Daily end-of-day S\&P/ASX 200 price index & DataStream & ASX200I \\
\hline Daily end-of-day S\&P/ASX 200 volatility index & DataStream & AXVIVOL \\
\hline Monthly Australian unemployment rate trend & DataStream & AU090144T \\
\hline Quarterly Australian gross domestic production (GDP) & DataStream & AUQL2104Q \\
\hline Bank Accepted Bills/Negotiable Certificates of Deposit - 1 m & RBA & 1-m BABs/NCDs \\
\hline OECD based Recession Indicators for Australia & FRED & AUSREC \\
\hline
\end{tabular}




\section{METHODOLOGY}

It is often noted that the Australian options market is illiquid (see O'Connell \& O'Grady (2007)). Furthermore, there is some evidence that in the Australian market, quarterly rebalancing periods offer better returns for Buy-Write strategies (see Mugwagwa et al. (2012)). Given that OTM put premiums are less expensive than OTM call premiums (evaluated at the same parameter values), to protect a portfolio at a certain level with reasonable cost, we buy put options with maturity of 6-months and write call options with a maturity of 3 months. We use two different models for valuing all portfolios.

Using Traded option market data (OMD model) and Using Black-Scholes option pricing formula (BSP model). The rebalancing dates are chosen with respect to the expiry dates of Australian option market, as shown in Figure 17, Appendix. In this study, we examine the performance of holding the S\&P/ASX 200 index with active, passive and zero-cost collars as well as protective put strategies over the period of January 2008 to March 2016. January 2008 coincides with the launch of the S\&P/ASX 200 Volatility Index. We require this Index to enable the implementation of the active collar strategies as well as for the calculation of the Black-Scholes option prices.

The strategies investigated in this paper are listed in Table 2, along with the corresponding call write ratio per index unit and the percentages of moneyness for the call and put options. Static strategies have a one-to-one call write ratio per unit of index and various degrees of moneyness of the OTM calls and OTM puts. The two protective put strategies as portfolio insurance, consist of long positions on the index and long positions on OTM puts with various moneyness. For these strategies, the number, NP of put options are calculated via

$N^{P}=\frac{V}{S * M}$

here $V$ is the investment amount of the current period, $\mathrm{S}$ is the current index level and $\mathrm{M}$ is the S\&P/ASX 200 index multiplier which is 10 (p. 11, ASX (2008))3. The zero-cost collar strategies are protective puts with the addition of as many short OTM call options required to cover the cost of the put options. The call and put options used in the zero-cost collar strategies are of six months maturity. Active strategies and the new strategy are explained in details Section 3.1 and Section 3.2.

TABLE 2

INSPECTED STRATEGIES

\begin{tabular}{|l|c|c|c|c|}
\hline Notation & Type & Call Write Ration & \% OTM Call & \% OTM Put \\
\hline Index & naked & NA & NA & NA \\
\hline$S_{1}$ & static collar & 1 & 7 & 5 \\
\hline$S_{2}$ & static collar & 1 & 5 & 5 \\
\hline$S_{3}$ & static collar & 1 & 5 & 2 \\
\hline$S_{4}$ & static collar & 1 & 2 & 2 \\
\hline$S_{5}$ & static collar & 1 & 4 & $2-4$ \\
\hline$P P_{1}$ & protective put & NA & NA & $5-7$ \\
\hline$P P_{2}$ & protective put & NA & NA & 2 \\
\hline$Z C_{1}$ & zero-cost collar & covers the put cost & 2 & 3 \\
\hline$Z C_{2}$ & zero-cost collar & covers the put cost & 3 & varied, base 2 \\
\hline$A_{1}$ & active collar & varied & varied, base 2 & varied, base 2 \\
\hline$A_{2}$ & active collar & varied & varied, base 3 & varied, base 2 \\
\hline$A_{3}$ & active collar & varied & varied, base 4 & varied, base 3 \\
\hline$A_{4}$ & active collar & varied & varied, base 2 & varied, base 3 \\
\hline$A_{5}$ & active collar & varied & varied, base 3 & varied, base 3 \\
\hline$A_{6}$ & active collar & varied & varied, base 4 & varied \\
\hline$N Z$ & active collar & varied & varied & \\
\hline
\end{tabular}




\section{Active Collar Strategy}

The active collar strategies, which are based on the trading rules of Szado \& Schneeweis (2010a), require computing the following quantities: The moneyness of the OTM call options (\% OTM Call), the moneyness of the OTM put options (\% OTM Put), and the call write ratio (CWR) which is the ratio at which call options are written per unit of index. The puts options are always purchased in the ratio of 1: 1 with the index.

To compute the CWR and the moneyness of OTM call and OTM put options, Szado \& Schneeweis $(2010 a)$ used three market signals: a volatility signal ( $\mathrm{vol})$, a momentum signal $(\mathrm{m})$ and a macroeconomic signal $(e)$, using the following formulae:

$$
\begin{gathered}
\text { CWR }=1+0.25 \operatorname{Sig}_{T}^{\text {vol }} \\
\% \text { OTM Call }=(\text { Call Base })+\operatorname{Sig}_{T}^{m}+\operatorname{Sig}_{T}^{e} \\
\% \text { OTM Put }=(\text { Put Base })+\text { Sig }_{T}^{m}+\text { Sig }_{T}^{e}
\end{gathered}
$$

where the base rates of the call and put options for the different active strategies are chosen as shown in Table 2. The usage and the computation of the volatility, momentum and macroeconomic signals are listed in Table 3.

TABLE 3

THE THREE MARKET

\begin{tabular}{|c|c|c|}
\hline Signals & Used For & Calculation \\
\hline Volatility: Sig $_{T}^{\text {vol }}$ & CWR & $T$-day SMA of VIX \\
\hline Momentum: Sig $_{T}^{m}$ & \% OTM Call and \% OTM Put & $T$-day SMA of the Index \\
\hline Macroeconomic: Sig $_{T}^{e}$ & \% OTM Call and \% OTM Put & $T$-day SMA unemployment rate \\
\hline
\end{tabular}

The three market signals; notations, purpose, and computations. Here SMA represents a simple moving average, VIX the S\&P/ASX 200 volatility index and Index the S\&P/ASX 200 index. We use $\mathrm{T}=50$ days for short term, $\mathrm{T}=150$ days for medium term and $\mathrm{T}=200$ days for long term averages.

The mathematical formulation of the volatility signal, momentum signal and macroeconomic signal are briefly outlined below (for detailed explanations and examples, see Szado \& Schneeweis (2010a)). Let $V_{0}$ be the Spot VIX (Spot VIX) being in the range of one-standard-deviation away from the T-day SMA of VIX implies the medium level of anxiety in the market; being in the lower tail indicates a low level of anxiety of the market; being in the upper tail indicates a high level of anxiety of the market. The volatility signal is

$$
\operatorname{Sig}_{T}^{\mathrm{vol}}=\left\{\begin{array}{ccc}
1 & \text { when } V_{0} \leq \mu_{T}^{V}-\sigma_{T}^{V} & \text { (low level of anxiety in the market) } \\
0 & \text { when } V_{0} \in\left(\mu_{T}^{V}-\sigma_{T}^{V}, \mu_{T}^{V}+\sigma_{T}^{V}\right) & \text { (medium level of anxiety in the market ) } \\
-1 & \text { when } V_{0} \geq \mu_{T}^{V}+\sigma_{T}^{V} & \text { (high level of anxiety in the market) }
\end{array}\right.
$$

This is used to establish the call write ratios so that

$$
C W R= \begin{cases}1.25 & \text { when the market anxiety level is low } \\ 1 & \text { when the market anxiety level is medium } \\ 0.75 & \text { when the market anxiety level is high }\end{cases}
$$


The momentum signal, $\mathrm{Sig}_{T}^{\mathrm{m}}$ is used to determine whether the index is experiencing an upward or downward phase. A call writer prefers that the options not be exercised and so chooses a strike level higher than the expected index level. The momentum signal adds $1 \%$ to the moneyness of the options in the case of an expected upward trend, subtracts $1 \%$ from the moneyness of the options in the case of an expected downward trend, and is formulated as:

$$
\operatorname{Sig}_{T}^{m}=\left\{\begin{array}{c}
1 \quad \text { when } I_{w} \geq \mu_{T}^{I} \\
-1 \quad \text { when } I_{w}<\mu_{T}^{I}
\end{array}\right.
$$

Here $\mu_{T}^{I}$ denotes the T-days simple moving average of S\&P/ASX 200 index and $I_{w}$ the 5 days moving average of the index. For the momentum signal, we adopt that $\mathrm{T}=150$ for call options and $\mathrm{T}=200$ for put options.

For the calculation of their macroeconomic signal, Szado \& Schneeweis (2010a) adapted the work of Boyd et al. (2005) who examined the effect of the unemployment rate on the market during expansionary and recession periods. They found that in expansionary periods, stocks typically rise on bad unemployment news, while the opposite relationship holds for contraction periods. To reflect this behavior, the moneyness percentage of OTM calls increases and the percentage for OTM puts decreases when the unemployment is high during expansionary economic periods. Hence Szado \& Schneeweis (2010a) incorporate the macroeconomic signal as multiplication of the business cycle indicator and the unemployment rate indicator:

$$
\operatorname{Sig}_{T}^{e}=M_{e, T} M_{U, T}
$$

where the business cycle indicator $M_{e, T}$ is 1 for expansionary economic periods and -1 for recession periods.

The unemployment rate indicator $M_{U, T}$ is given by

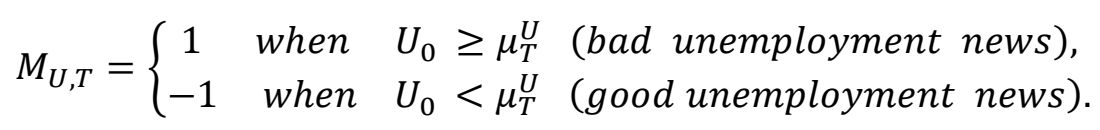

Here $U_{0}$ is the current unemployment rate ${ }^{4}$, and $\mu_{T}^{U}$ is the T-days simple moving average of unemployment rate. We utilize three months moving average of unemployment rate for call options and six months moving average for put options, being consistent with their maturity. During the expansionary economic period, if the current unemployment rate is greater than the last $\mathrm{T}$-months moving average of unemployment rates then it would be considered to be bad news and it is expected that stocks will rise. In these circumstances we prefer, as a call writer, a higher OTM strike level for the call options, and as a put buyer a higher OTM strike level for the put options.

\section{New Active Collar Strategies}

The volatility (using VIX) signal in the active strategies introduced by Szado \& Schneeweis (2010a) does not reflect the magnitude of the volatility, because the current volatility could be high but still lie within the one standard deviation range of $\mathrm{T}$-days simple moving average. In that case, high volatility indicates a high level of anxiety in the market, but the signal only indicates a medium level of anxiety. We propose a new active strategy $(N S)$ that exploits the magnitude of the volatility. Specifically, we propose using a set of volatility ranges in additions to the volatility signal. We consider the four volatility ranges, $V R_{i}, \mathrm{i}=1,2,3,4$ :

$$
\begin{array}{rlrl}
0 & <V R_{1} \leq 0.12 & 0.12 & <V R_{2} \leq 0.2 \\
0.2 & <V R_{3} \leq 0.3 & 0.3 & <V R_{4} \leq 1
\end{array}
$$


Here the volatilities are per annum. We favor these ranges by inspecting the history of the CBOE VIX to 2008, see Figure 1. If the current volatility is in the range $V R_{1}$ then the investor may reap the highest possible profit by writing OTM calls close to being at-the money (ATM) and buying the OTM puts close to being ATM to profit from the small movement of the index. The volatility range, $V R_{4}$ represents extreme events in the market, such as a financial crisis or a market bubble. The volatility in the range $V R_{2}$ is consistent with a medium level of anxiety and $V R_{3}$ with a high level of anxiety in the market.

\section{FIGURE 1 \\ S\&P/ASX 200 VIX INDEX DURING THE ENTIRE PERIOD 2008-2016}

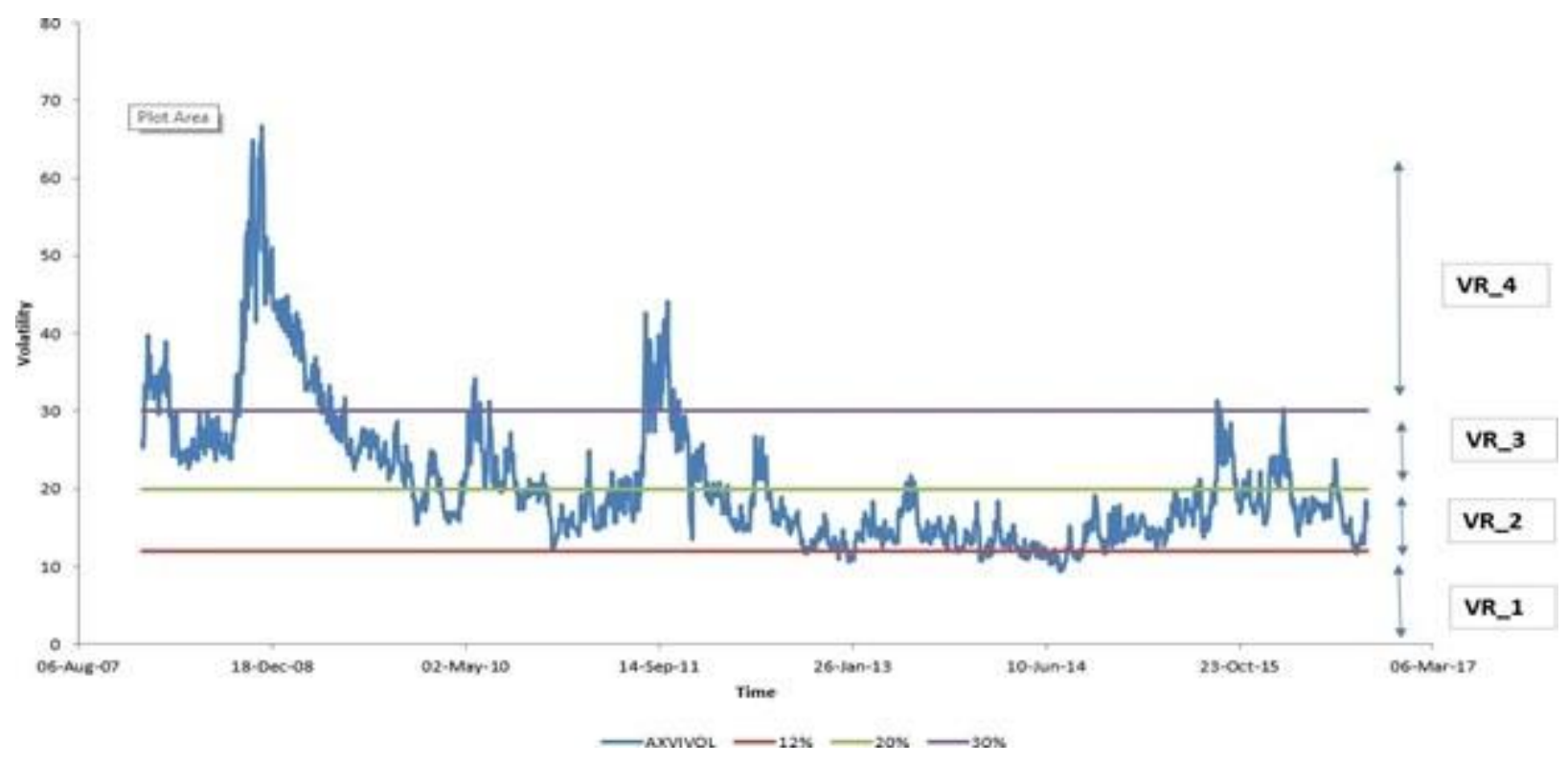

We make use of both short and medium term momentum signals for call options with 3-months maturity to capture the persistence of the movement. We consider, for example, that an increase in the index is more likely if both short term and the medium-term momentum signals are one. We summarize our interpretation of the momentum signals as follows:

TABLE 4

\section{MARKET BEHAVIOR ASSUMPTION}

\begin{tabular}{|r|r|l|}
\hline $\mathrm{T}_{1}$ & $\mathrm{~T}_{2}$ & Interpretation \\
\hline 1 & 1 & increase in the index is more likely \\
\hline-1 & -1 & decrease in the index is more likely \\
\hline-1 & 1 & decrease in the index is more likely \\
\hline 1 & -1 & increase in the index is more likely \\
\hline
\end{tabular}

where $T_{1}=50, T_{2}=150$ and $T_{3}=250$ are moving average windows. We used the short term $\left(T_{1}=50\right)$ and the medium term $\left(T_{2}=150\right)$ momentum signal for call options with the 3-months maturity.

Table 5 shows the call write ratios for the new strategy with respect to the volatility range. 
TABLE 5

CALL WRITE RATIO (CWR) WITH RESPECT TO VOLATILITY RANGES

\begin{tabular}{|r|r|r|r|}
\hline$V R_{1}$ & $V R_{2}$ & $V R_{3}$ & $V R_{4}$ \\
\hline 1 & 0.75 & 0.5 & 0.25 \\
\hline
\end{tabular}

The moneyness of call options, based on volatility range and momentum signal at the current rebalancing period are given in Table 6.

TABLE 6

THE MONEYNESS PERCENTAGE FOR OTM CALL OPTIONS

\begin{tabular}{|r|r||r|r|r|r|}
\hline $\mathrm{T}_{1}$ & $\mathrm{~T}_{2}$ & $V R_{1}$ & $V R_{2}$ & $V R_{3}$ & $V R_{4}$ \\
\hline 1 & 1 & 3 & 5 & 6 & 7 \\
\hline-1 & -1 & 2 & 4 & 5 & 7 \\
\hline-1 & 1 & 2 & 4 & 5 & 7 \\
\hline 1 & -1 & 3 & 5 & 6 & 7 \\
\hline
\end{tabular}

The moneyness of OTM put options to buy is always $2 \%$.

\section{RESULTS}

We compute returns using period by period portfolio balance where the portfolio consists of various components of strategies, including equity and option. In addition to the standard measures, our performance analysis relies on the Adjusted Sharpe ratio, the Excess Return Entropy ratio, and the Stutzer Index. The Adjusted Sharpe ratio accounts for skewness and kurtosis. The Excess Return Entropy ratio is computed by replacing the standard deviation by the Shannon entropy in the Sharpe ratio. The entropy measure accounts for higher moments of a distribution and consequently offers a better explanation of the distribution (see Ebrahimi et al. (1999), Milev et al. (2012)). The Stutzer index is the rate at which the probability of under-performance approaches zero. The highest the Stutzer index indicates the best investment.

We compute the annual statistics for the returns of each strategy: Mean, Cumulative Return at the end of the holding period, Standard Deviation, Semi Devia tion, Maximum Return, Minimum Return, Skewness, Excess Kurtosis. In addition, we compute the performance measures: Adjusted Sharpe Ratio, Excess Return Entropy ratio and the Stutzer Index. The formulae for these performance measures are given in Appendix. The tables of the statistics and the performance measures for all portfolios and all periods are placed in Appendix.

\section{The Results for Entire Period 2008-2016}

Figure 2 shows cumulative returns of the index. The index value is below the initial investment value during most of the holding period and is almost in neutral position in the end of the holding period. 


\section{FIGURE 2 \\ GRAPH OF CUMULATIVE RETURNS OF THE INDEX FOR THE ENTIRE PERIOD 2008-2016}

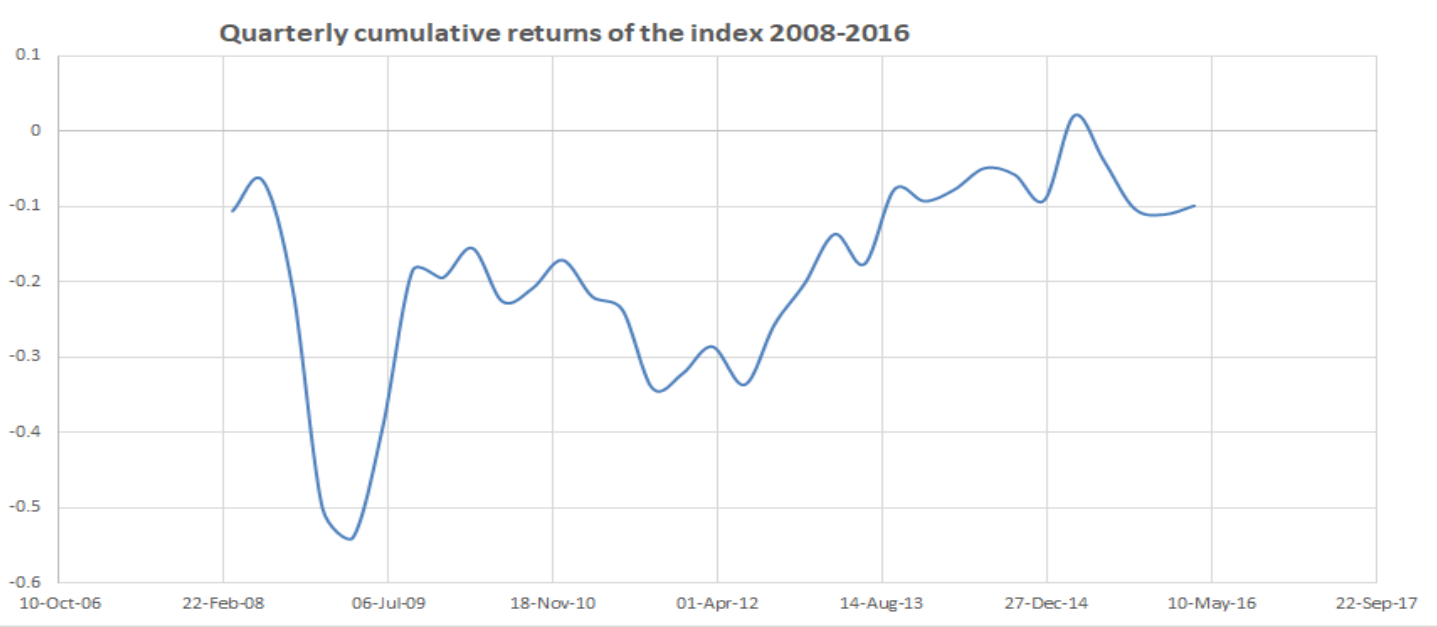

Figure 3 summarizes the three best performing portfolios for the option market data model and for the Black-Scholes Option Pricing model under the performance measures; Adjusted Sharpe Ratio ("AdjSha"), Excess Return -Entropy ratio ("ExRetEnt") and Stutzer Index ("Stut”).

FIGURE 3

THE THREE BEST PERFORMING PORTFOLIOS FOR THE ENTIRE PERIOD 2008-2016

\begin{tabular}{|c|c|c|c|c|c|c|}
\hline 2008-2016 & \multicolumn{3}{|c|}{ Option Market Data Model } & \multicolumn{3}{c|}{ Black-Scholes Pricing Model } \\
\hline AdjSha & S4 & S5 & S3 & S5 & S3 & S4 \\
\hline ExRetEnt & PP1 & ZC1 & NS & PP1 & NS & ZC1 \\
\hline Stut & ZC1 & PP1 & NS & ZC1 & PP1 & ZC2 \\
\hline
\end{tabular}

Note that the accuracy of the performance measure Adjusted Sharpe ratio during this holding period is debatable, as the data contains the extreme market event, GFC. Under the performance measure ExRetEnt and Stut, for the option market data model, we found that the protective put portfolio, $P P_{1}$ and Zero-cost collar portfolio, $Z C_{1}$ outperforms all the collar strategies, and the best performing collar strategy is the new strategy. The out-performance of the protective put can be attributed to absence of the cost of exercise for the written call options. We also remark that the protective put portfolio, $P P_{1}$ with $2-4 \%$ OTM put outperforms the protective put portfolio, $P_{2}$ with 5-7\% OTM put. Even though the idea for collar is to mitigate the cost of the protective put by writing call options, the risk involved appeared to be higher than what is expected, particularly during and after extreme market events like GFC. As mentioned, unexpected sharp increases in the index after the GFC caused large exercise expense for the written call options, the loss at one such quarter reached more than $10 \%$ of the initial investment amount for most of our portfolios (see Appendix for the percentages of call exercise cost over the holding period to the initial investment for all collar portfolios). Zero-cost collar portfolio reflects the market conditions, as it captures the parity between call and put options in the market. Out-performance of the new strategy is due to the adjustment of the magnitude of the volatility in the trading strategy. However, the new strategy was not immune the call exercise losses, occurred as a result of unusual increase in index. We note that there is some discrepancy 
between the results using the option market data model (say OMD model) and those using Black-Scholes pricing model (say BSP model) for the entire period 2008-2016.

A comparison graph of performance measures applied to portfolios for OMD and BSP models over the entire period 2008-2016 are given in Figures 4 and 5. The results are ordered with respect to Stutzer index. We normalized some values for visualization by multiplying the entire measure (except Stutzer index) by a constant.

FIGURE 4

COMPARISON OF PERFORMANCE MEASURES APPLIED TO PORTFOLIOS FOR OMD MODEL OVER 2008 - 2016

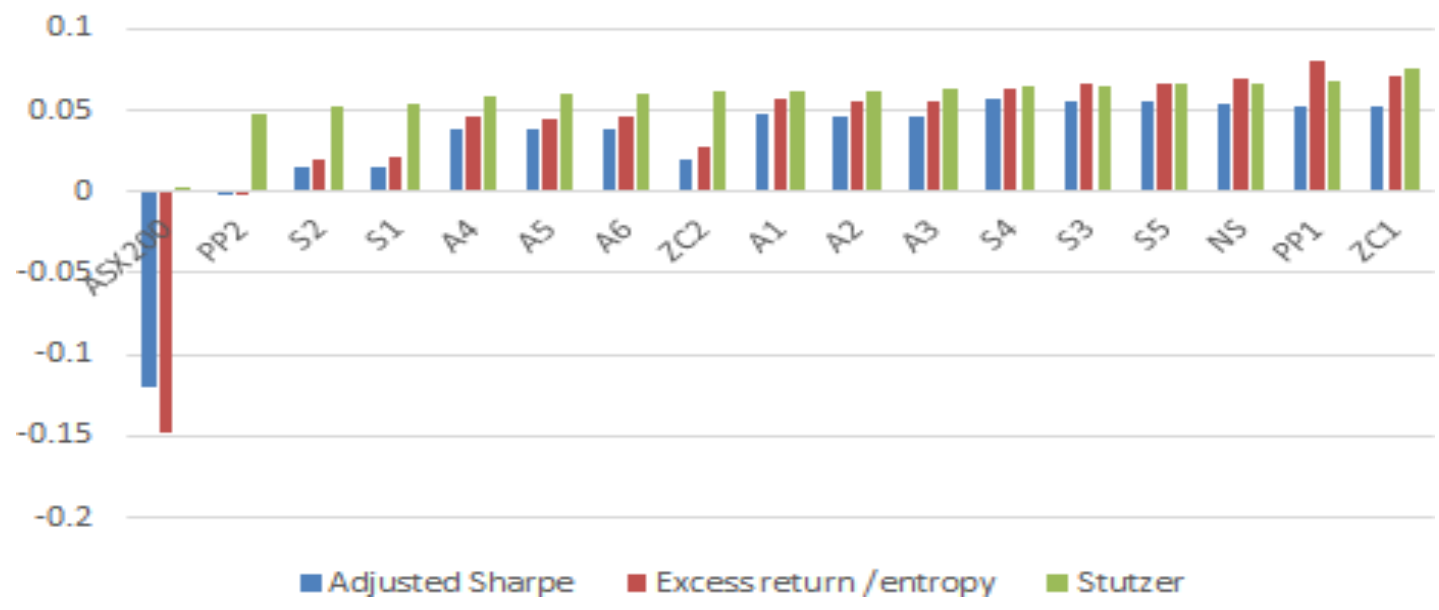

FIGURE 5

COMPARISON OF PERFORMANCE MEASURES APPLIED TO PORTFOLIOS FOR BSP MODEL OVER 2008 - 2016

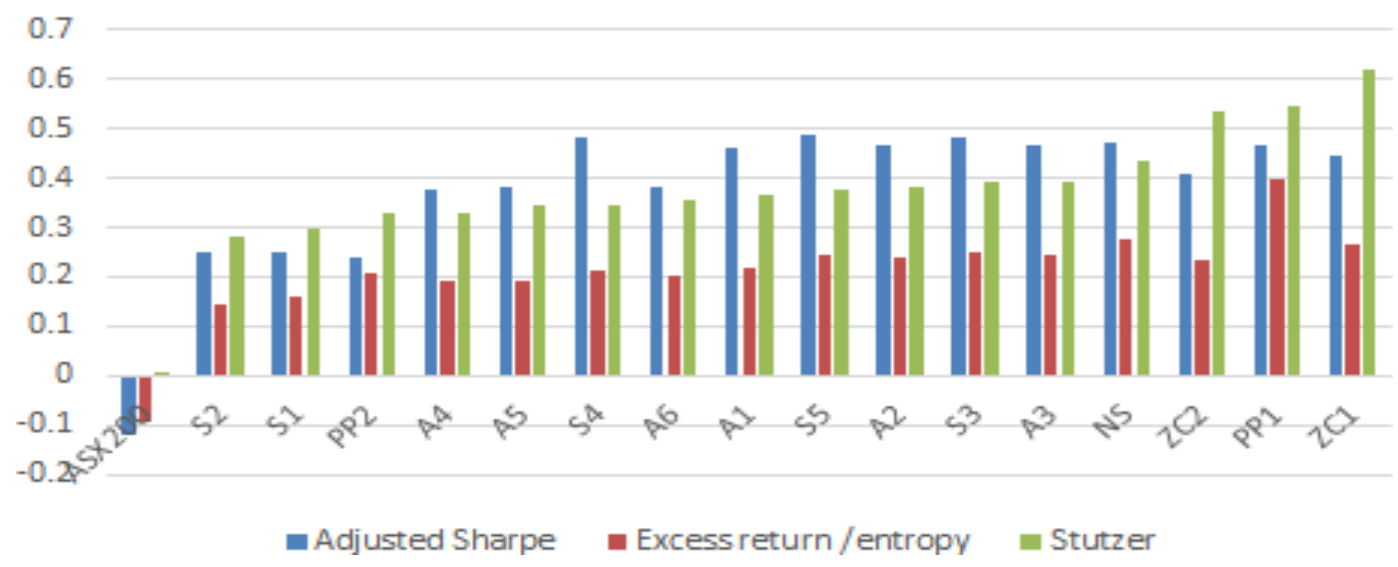

The Results for Subperiods 2008-2012, 2010-2014 and 2012-2016

Figure 6 summarizes the three best performing portfolios for the OMD model and for the BSP model for each subperiods. Appendix contains the graphs of comparison of performance measures applied to all portfolios for each subperiods. The tables of statistics and performance results for all subperiods are in Appendix. 
FIGURE 6

THE THREE BEST PERFORMING PORTFOLIOS FOR ALL THE SUBPERIODS

\begin{tabular}{|c|c|c|c|c|c|c|c|c|c|c|c|c|c|c|c|c|c|c|}
\hline \multirow{3}{*}{$\begin{array}{c}\text { Subperiods } \\
\text { Models } \\
\text { AdjSha }\end{array}$} & \multicolumn{6}{|c|}{ 2008-2012 } & \multicolumn{6}{|c|}{ 2010-2014 } & \multicolumn{6}{|c|}{ 2012-2016 } \\
\hline & \multicolumn{3}{|c|}{ Market Data } & \multicolumn{3}{|c|}{ Black-Scholes Pricing } & \multicolumn{3}{|c|}{ Market Data } & \multicolumn{3}{|c|}{ Black-Scholes Pricing } & \multicolumn{3}{|c|}{ Market Data } & \multicolumn{3}{|c|}{ Black-Scholes Pricing } \\
\hline & PP1 & $\mathrm{A} 1$ & A3 & S5 & S3 & A3 & PP1 & A2 & A1 & A2 & A3 & A1 & A1 & $\mathrm{A} 2$ & A3 & NS & S3 & PP1 \\
\hline ExRetEnt & PP1 & A2 & S4 & NS & PP1 & S5 & PP1 & $\mathrm{ZC} 1$ & A1 & PP1 & A1 & A2 & $\mathrm{ZC} 1$ & $\mathrm{ZC2}$ & PP1 & PP1 & $\mathrm{ZC} 1$ & $\mathrm{ZC} 2$ \\
\hline Stut & A2 & A1 & PP1 & $\mathrm{ZC1}$ & NS & $\mathrm{ZC2}$ & PP1 & $\mathrm{ZC1}$ & $\mathrm{ZC} 2$ & $\mathrm{ZC1}$ & PP1 & $\mathrm{ZC2}$ & $\mathrm{ZC1}$ & NS & PP1 & $\mathrm{ZC1}$ & $\mathrm{ZC} 2$ & PP1 \\
\hline
\end{tabular}

Now we proceed by explaining specific market conditions for each subperiod and then analyzing of the results.

The subperiod 2008- 2012 is Global Financial Crisis period. The index drops below the initial investment value and the cumulative return is $-29 \%$ in the end of holding period (see Figure 7). Adjusted Sharpe Ratio, Excess Return -Entropy ratio and Stutzer Index are all negative in this subperiod for the OMD model. There was not enough time for the investment to recover during this holding period.

\section{FIGURE 7 \\ CUMULATIVE RETURNS OF THE INDEX FOR THE SUBPERIOD 2008-2012}

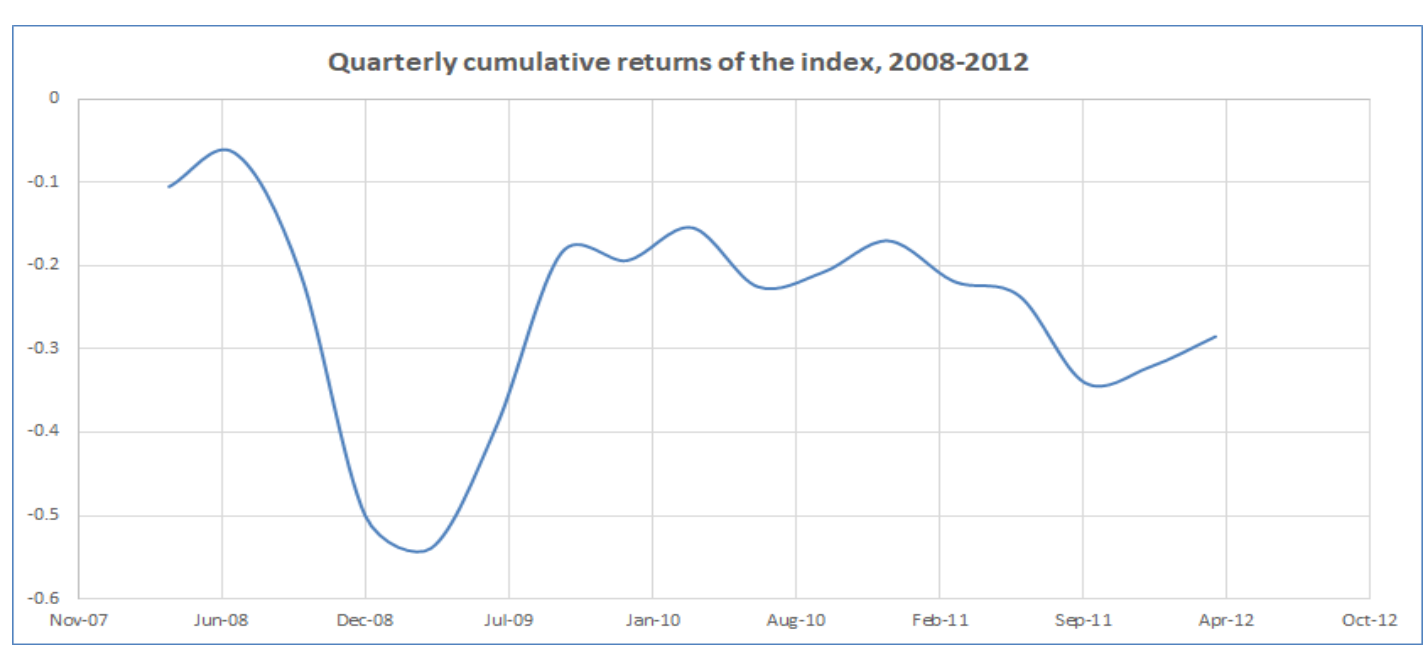

During such a turbulence period of the market, the market data model gives that the protective put portfolio $P P_{1}$ with 2\%-4\% OTM Put is the best strategy under AdjSha and ExRetEnt. The returns of the collar are not sufficient to justify the risk of the collar strategies during these extreme negative market conditions. Under the performance measure Stut, Active strategies with base 2\%-3\% OTM call and base $2 \%$ OTM put is the fastest recovering collar portfolio, moving from negative to positive returns. The new strategy portfolio appears among the best performing portfolios in the BSP model, but not in the OMD model. This is because the options with the OTM percentages, as suggested by the new strategy are not available in the market during the extreme events period. Furthermore, in this subperiod, the OMD model produces negative performance measures across all strategies, whereas the BSP model produces positive performance measures for all portfolios (except the index). Therefore, the differences between realized option prices and Black-Scholes option prices are not incremental.

During the subperiod 2010-2014, the quarterly returns of the index are negative 59\% of the time (see Figure 8 for quarterly cumulative returns). The market moves from bearish to bullish towards the end of the subperiod and all portfolios end this subperiod with positive cumulative returns. Adjusted Sharpe Ratio, Excess Return -Entropy ratio and Stutzer Index are negative for the index, but positive for all the other portfolios for both of our models. 


\section{FIGURE 8}

\section{CUMULATIVE RETURNS OF THE INDEX FOR THE SUBPERIOD 2010-2014}

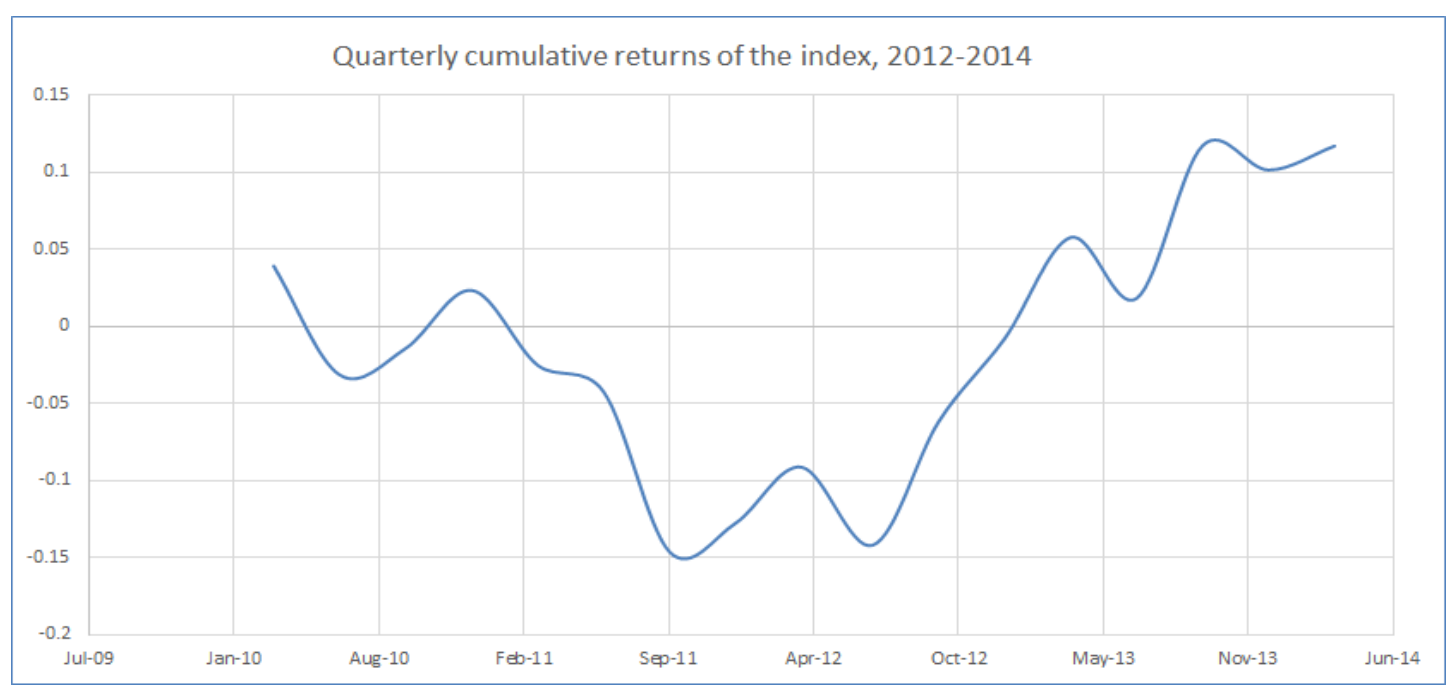

According to the OMD model, the protective put portfolio $P P_{1}$ with $2-4 \%$ OTM Put is the best performing portfolio under all performance measures. This shows that collar strategies contain more risk than their acceptable level relative to their returns during the bearish-to-bullish market period. The zerocost collar portfolio $Z C_{1}$ (2\% OTM Call and Put) is the best performing collar strategy under ExRetEnt and Stut. It somehow captured the market parity between covered calls and protective puts during the bearishto-bullish market period. The results of BSP model are again different from the results of the OMD model for this subperiod. However, both model show that the best performance portfolio is Protective put portfolio $P P_{1}$ under the performance measure ExRetEnt.

The quarterly returns of the index are positive 94\% of the time during the subperiod 2012-2016 (see Figure 9 for quarterly cumulative returns). The market is bullish. The index level increases $22 \%$ during this period.

FIGURE 9

CUMULATIVE RETURNS OF THE INDEX FOR THE SUBPERIOD 2012-2016

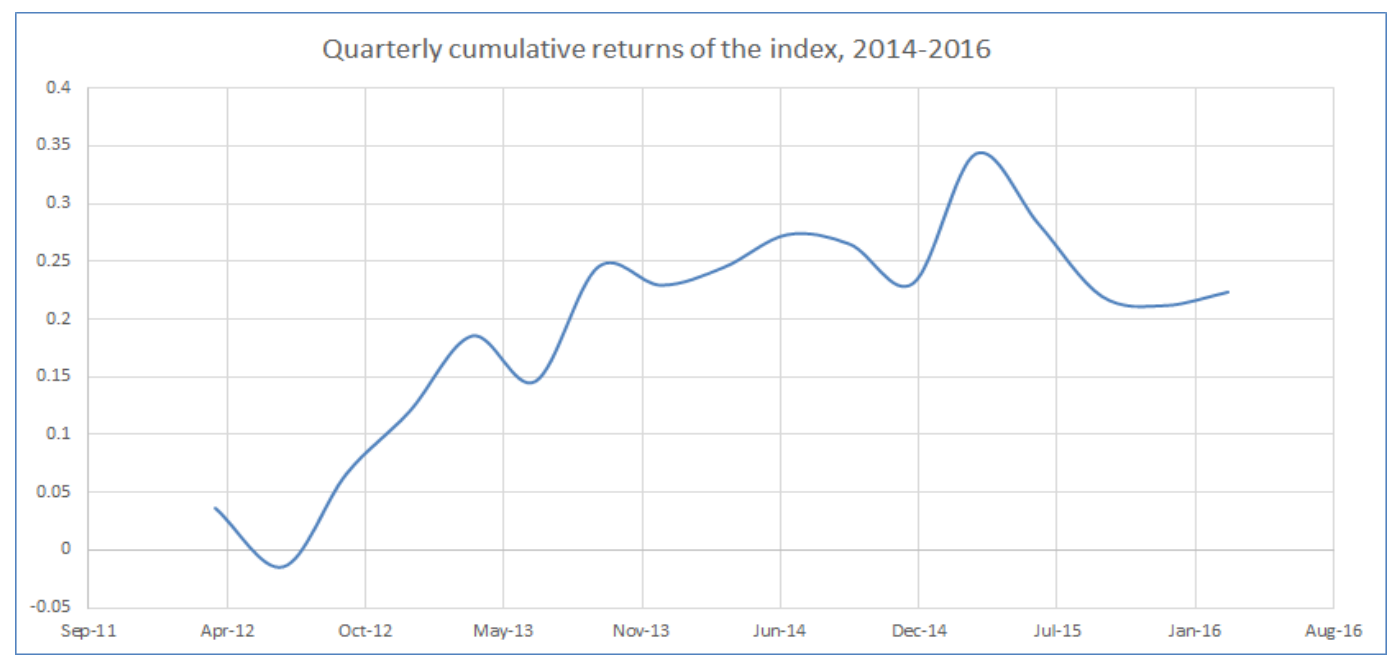


Since this subperiod is bullish, firstly, collar strategies involve higher risks. Secondly, the distribution of the index is far being normal, as the returns almost constantly increase. Therefore, the Adjusted Sharpe Ratio is not a reliable performance measure, particularly during this subperiod. The OMD model shows that, under the performance measures ExRetEnt and Stut, the best performing portfolio is the zero-cost collar portfolio $Z C_{1}$ ( $2 \%$ OTM Call and Put). The zero-cost collar portfolio $Z C_{2}$ (3\% OTM Call and Put), the new strategy portfolio and the protective put portfolio $P P_{1}$ with $2 \%$ OTM Put are among the best performing portfolios. outperformance under the performance measures ExRetEnt and Stut. Interestingly, the new strategy outperforms all the other collar strategies under the performance measures ExRetEnt and Stut. This is because accounting for the magnitude of the volatility in the new strategy reduces the risk of call exercise costs. The BSP model gives different results than the OMD model during this bullish subperiod as well. Both models agree that the best performance portfolio is the zero-cost collar portfolios, $Z C_{1}$ under the performance measure Stut.

\section{CONCLUSION}

We examined five static, two zero-cost, six active, one new collar and two protective put strategies varied in moneyness during the entire period 2008-2016 and the subperiods 2008-2012, 2010-2014 and 2012-2016. The performance of any strategy depends on the market conditions and how appropriate the strategy is, given the market movements. Under extreme market conditions, like Global Financial Crisis, protective put (with 2\% OTM put and 6-months maturity) should be preferred over any collar strategies in Australian market. The success of the protective put is shown to be due to its exemption from the exercise cost of the written call options in the collar strategies, particularly during the recovery period of the Global Financial Crisis of 2008-2009.

The risk of collar strategies is, in general, high, and even higher during the bullish periods of the market. The risk of call exercise losses should carefully be accounted for. The new strategy reduces the risk of collar by accounting the magnitude of the volatility during bullish periods. Even though complex strategies constructed by examining the market and economic signals (New and Active strategies) appear among the three best performing portfolios, Zero-cost collar strategy with 2\% OTM call and put, and 6-months maturity is preferable overall. During high level of anxiety in the market, such complex investment strategies would not work with high probability. Zero-cost collar strategies capture more information from the market than any complex collar strategy. The protective put strategies do not have the risk of call exercise costs as in collar strategies. It also appeared that the new strategy captures more information from the market than active strategies in general, due to the additional information used to account for the magnitude of volatility.

Furthermore, we showed that there are differences between the option market data model and the BlackScholes pricing model. The discrepancy is related to both the issues of the relative illiquidity of the option market in Australia and overpricing of the Black-Scholes option formula. Since the Australian market is illiquid, the option market data model may not find the option with required OTM level, while the BlackScholes pricing model assumes its existence. It should be kept in mind that the differences between the market option model and the Black-Scholes option pricing model are not incremental.

\section{ENDNOTES}

1. We also consider Leland ratio and stochastic dominance method which are often used for performance analysis of investments with non-normal return distributions, but we found them to be inappropriate for our study. The risk measurement of Leland (1999) assumes that the underlying market is normally distributed. According to our statistical tests, the returns of S\&P/ASX 200 index during the entire period 2008-2016 and subperiods 2008-2012, 2010-2014 and 2012-2016 are not normally distributed. Thus, the Leland ratio was not appropriate for our performance analysis. Given the large number of portfolios studied, applying stochastic dominance method together with Kolmogorov-Smirnov non-parametric test would be too complex. 
2. To determine the expansion and contraction business cycle periods in Australia, we use the Australian gross domestic production (GDP) year on year growth rate announced monthly (DataStream code: AUQL2104Q).

We apply back-testing on the data obtained from FRED for the period 20042014 and found GDP growth of $2.95 \%$ as the critical value: if the GDP growth is less than $2.95 \%$ then the Australian business cycle is generally in a contraction period.

3. The formula normally contains as a factor, the beta of the underlying. The beta of S\&P/ASX 200 index is assumed to be one.

4. Szado \& Schneeweis (2010a) uses a one-week simple moving average of unemployment rate, however, in Australia the minimum data frequency for unemployment rates is monthly so we use the current employment rate and monthly rather than weekly moving averages.

\section{REFERENCES}

Abid, F., Mroua, M., \& Wong, W.K. (2007). The impact of option strategies in financial portfolios performance: Mean variance and stochastic dominance approaches. SSRN Electronic Journal. id: 982332.

ASX. (2008). Introduction to index futures and options. Retrieved from http://www.asx.com.au/documents/products/intro_to_index_futures_and_options.pdf

Bacon, C.R. (2013). Practical Risk-Adjusted Performance Measurement. Wiley Publications.

Bookstaber, R., \& Clarke, R. (1985). Problems in evaluating the performance of portfolios with options. Financial Analyst Journal, 41(1), 48-62.

Boyd, H., Hu, J., \& Jagannathan, R. (2005). The stock market's reaction to unemployment news: Why bad news is usually good for stocks. Journal of Finance, 60(2), 649-672.

CBOE. (2012). Why should use equity collars? Retrieved from http://www.cboe.com/strategies/pdf/equitycollarstrategy.pdf

DJI. (2017). S\&P Dow Jones Indices, S\&P/ASX 200 VIX Methodology. Retrieved from http://us.spindices.com/indices/strategy/sp-asx-200-vix

Dublin, R. (2010). The varying cost of options and implications for choosing the right strategy. Journal of Financial Planning, pp. 62-70.

Ebrahimi, N., Maasoumi, E., \& Soofi, E. (1999). Ordering univariate distributions by entropy and variance. Journal of Econometrics, 90(2), 317-336.

El-Hassan, N., Hall, T., \& Tulunay, I. (2019). Performance of a balanced portfolio with active coveredcall strategies. Submitted for publication.

Figelman, I. (2008). Expected return and risk of covered call strategies. Journal of Portfolio Management, pp. 81-95.

Israelov, R., \& Nielsen, L.N. (2015). Covered calls uncovered. Financial Analysts Journal, 71(6), 44-57.

Israelov, R., \& Klein, M. (2016). Risk and return of equity index collar strategies. The Journal of Alternative Investments, 19(1), 1-14.

Israelov, R., Nielsen, L.N., \& Villalon, D. (2017). Embracing downside risk. The Journal of Alternative Investments, 19(3), 1-9.

Lassance, N., \& Vrins, F. (2017). Optimal R'enyi entropy portfolios. ArXiv Quantitative Finance, Portfolio Management. Retrieved from https://arxiv.org/abs/1705.05666v3

Leggio, K.B., \& Lien, D. (2008). Leland model more accurately evaluates efficacy of portfolio hedging strategies. Journal of Financial Planning, 21(1), 40-47.

Leland, H.E. (1999). Beyond mean-variance: Performance measurement in a non-symmetrical world. Financial Analysts Journal, 55(1), 27-36.

Milev, M., Inverardi, P.N., \& Tagliani, A. (2012). Moment information and entropy evaluation for probability densities. Applied Mathematics and Computation, 218, 5782-5795.

Mugwagwa, T., Ramiah, V., Naughton, T., \& Moosa, I. (2012). The efficiency of the buy-write strategy: Evidence from Australia. Journal of International Financial Markets, Institutions and Money, 22, 305-328. 
O'Connell, D., \& O'Grady, T.B. (2007). The buy-write strategy, index investment and the efficient market hypothesis: More Australian evidence. School of Economics and Finance Working Paper. Curtin University of Technology, School of Economics and Finance.

Ormos, M., \& Zibriczky, D. (2014). Entropy-based financial asset pricing. Plos One, pp. 1-21.

Philippatos, G.C., \& Wilson, C.J. (1972). Entropy, market risk and the selection of efficient portfolios. Applied Economics, 4(3), 209-220.

Stutzer, M. (2000). A portfolio performance index. Financial Analysts Journal, 56(3), 52-61.

Szado, E., \& Kazemi, H. (2009). Collaring the cube: Protection options for qqq etf portfolio. The Journal of Alternative Investments, 11(4), 24-42.

Szado, E., \& Schneeweis, T. (2010a). Loosening your collar: Alternative implementation of QQQ collars. Journal of Trading, 5(2), 35-56.

Szado, E., \& Schneeweis, T. (2010b). Risk mitigating collar strategy: Loosening your collar: Alternative implementation of $Q Q Q$ collars. Summary version. OIC.

Usta, I., \& Kantar, Y.M. (2011). Mean-variance-skewness-entropy measures: A multi-objective approach for portfolio selection. Entropy, 13, 117-133.

\section{APPENDIX}

\section{Statistical Tables and Performance Measures}

Tables in this section present our results, as annual statistics: Mean, Cumulative Return (CumRet) at the end of holding period, Standard Deviation (StDev), Semi-Deviation (SemiDev), Maximum Return (MaxRet), Minimum Return (MinRet), Skewness (Skew), Excess Kurtosis (ExKurt), and performance measures: Adjusted Sharpe Ratio (AdjSha), Excess Return -Entropy ratio (ExRetEnt) and Stutzer Index (Stut). The brief description of the strategies is given in Table 2. Section 3 explains the methodology for the construction of the portfolios. 


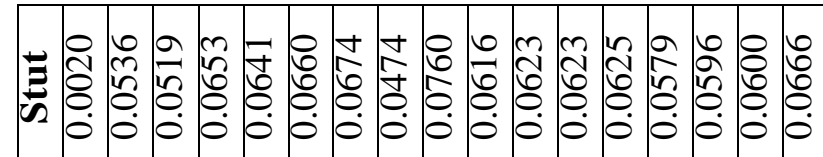




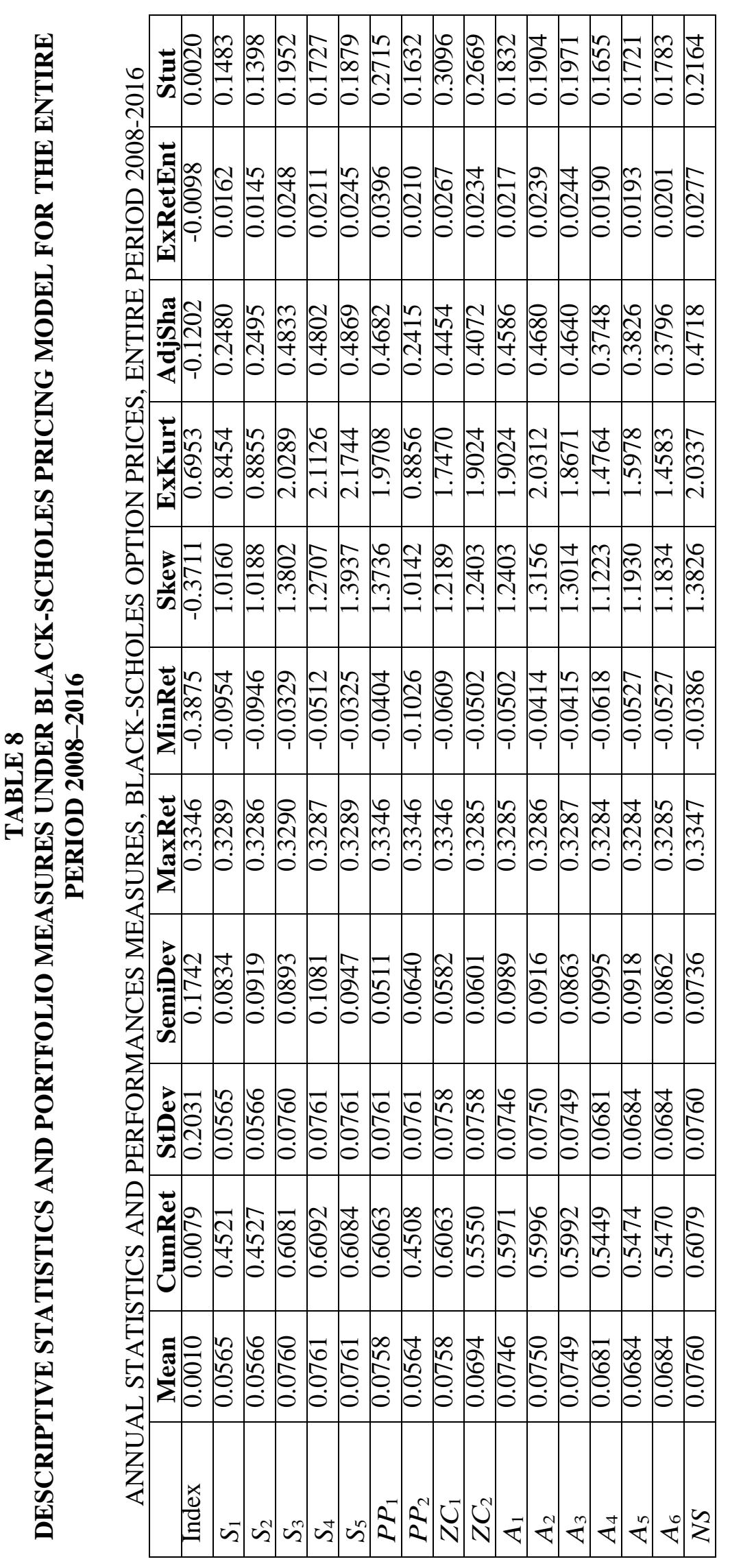




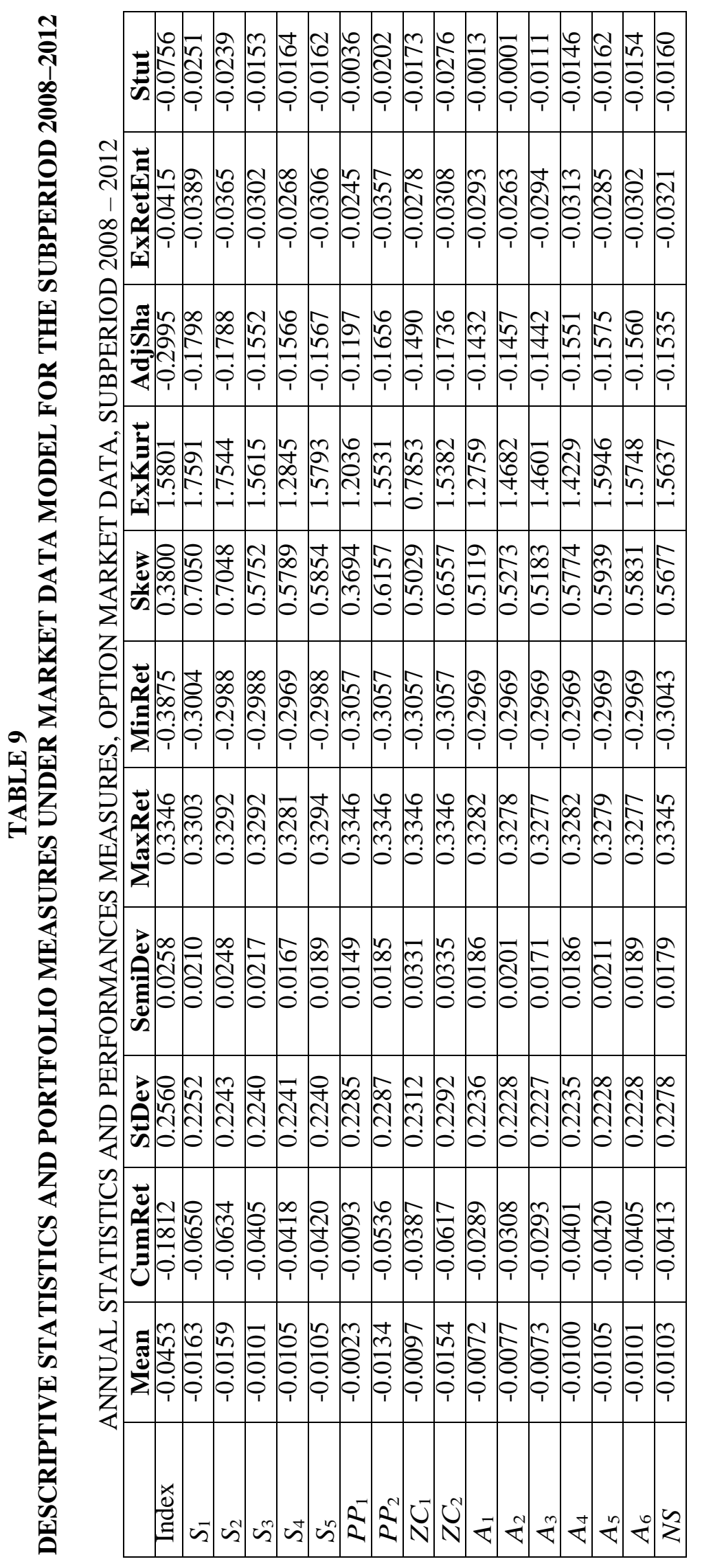

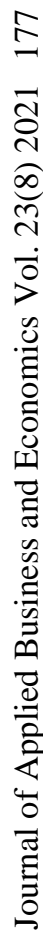




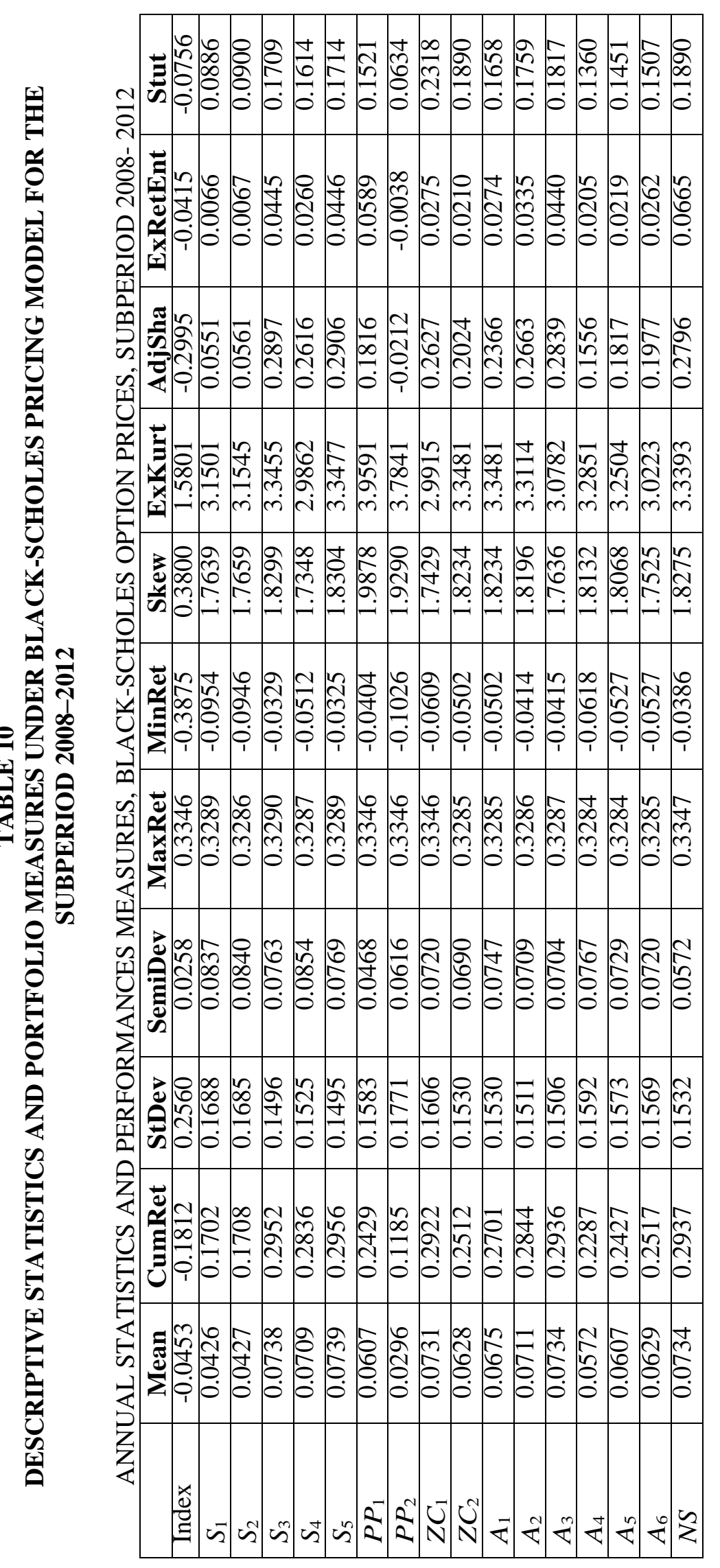

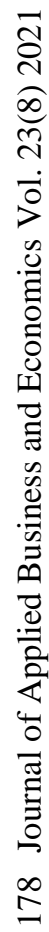




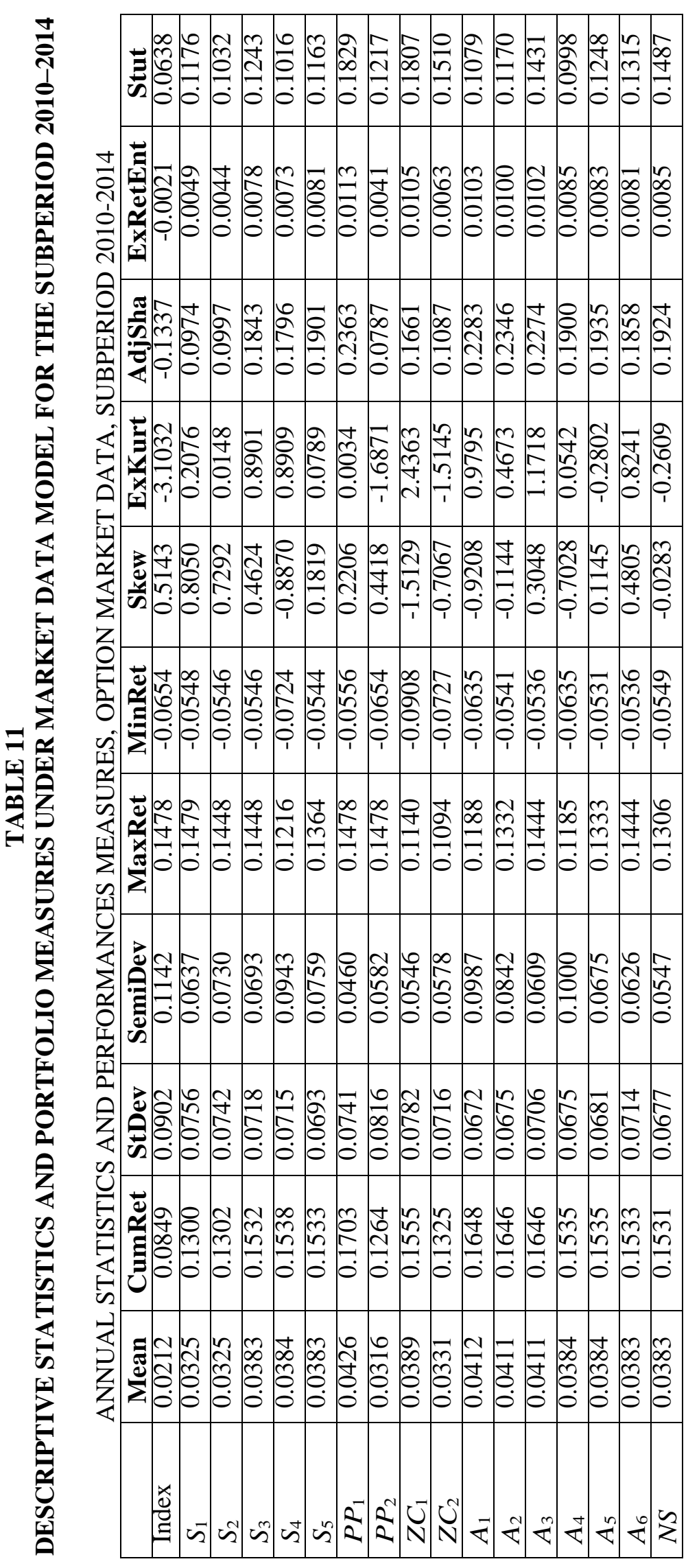




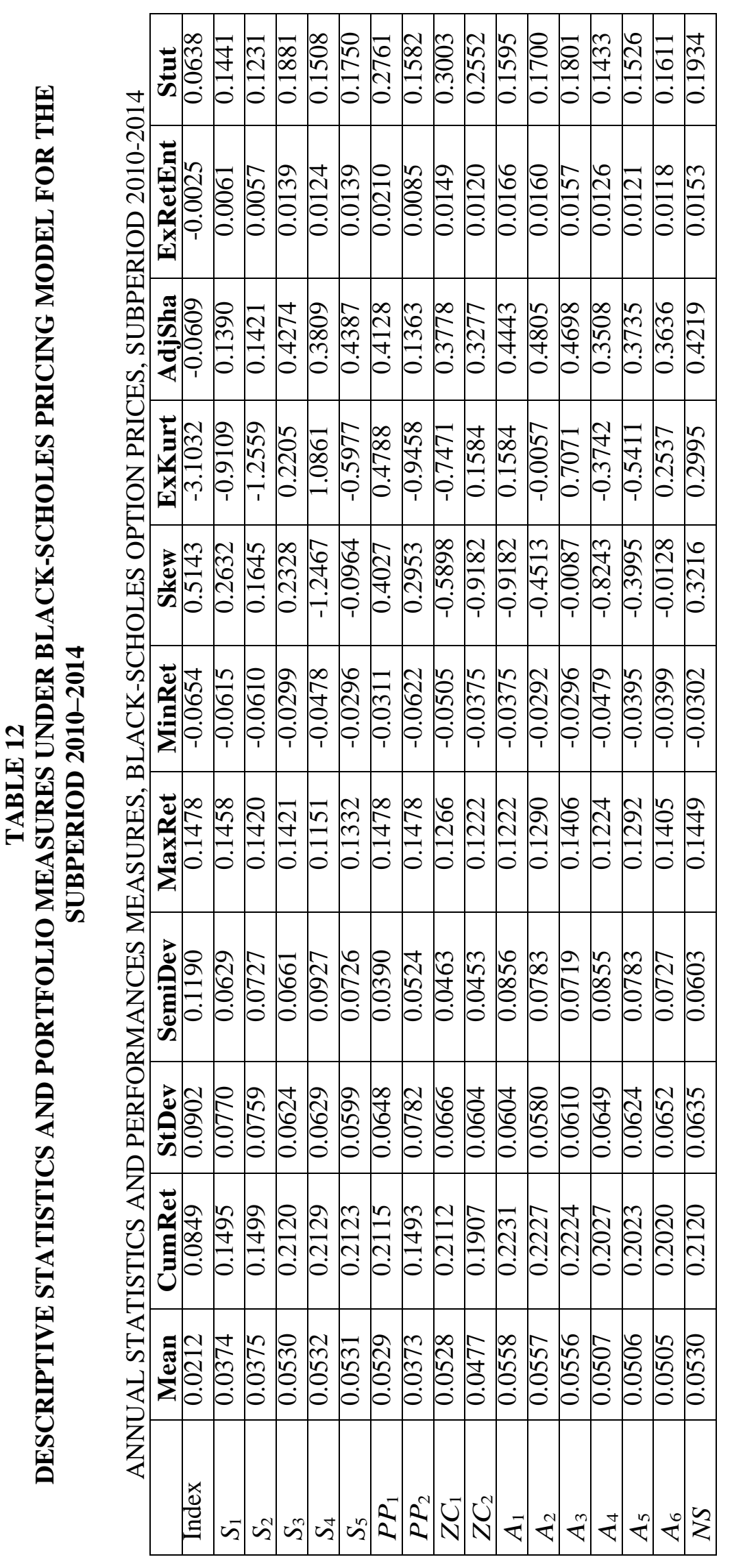




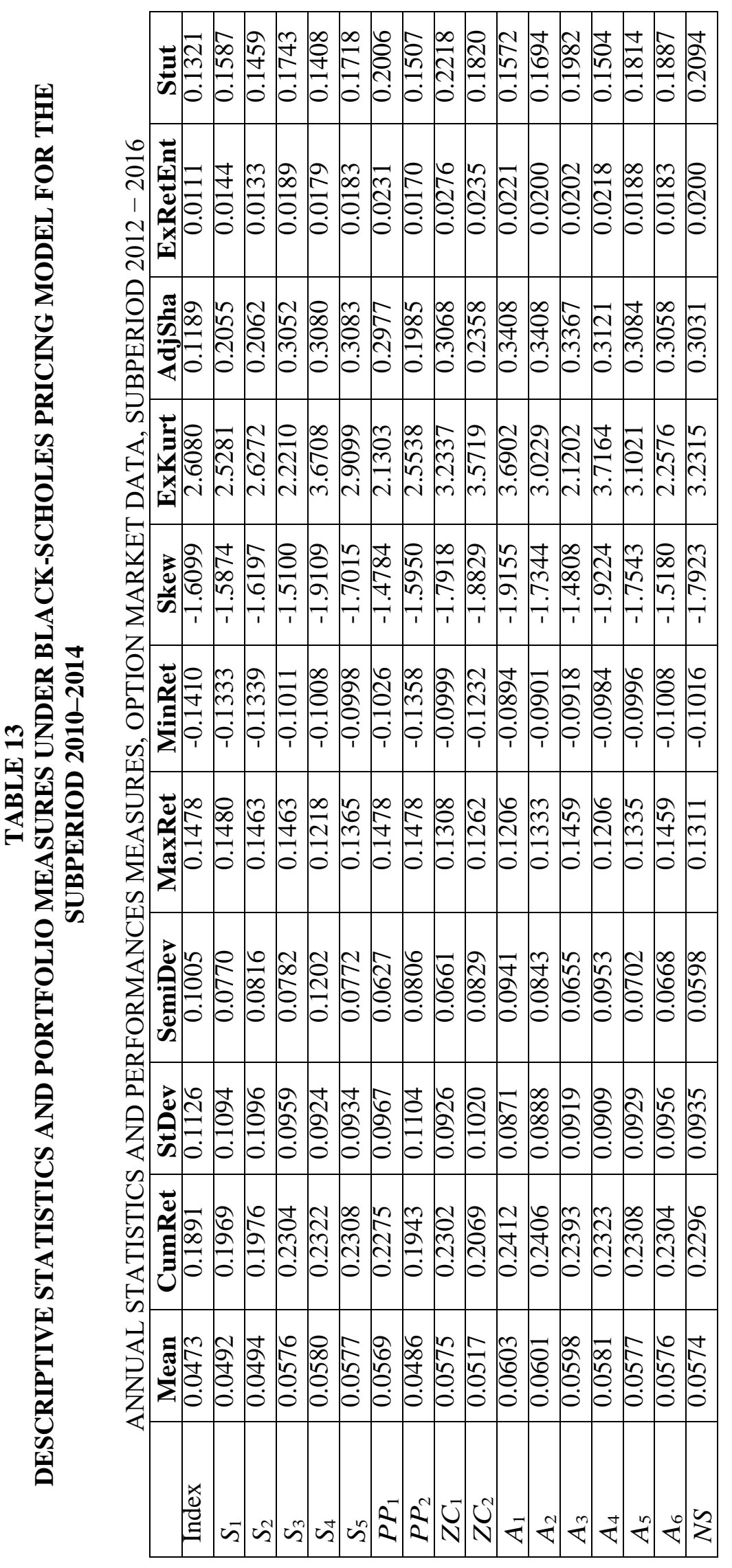

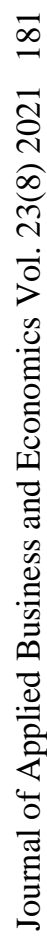




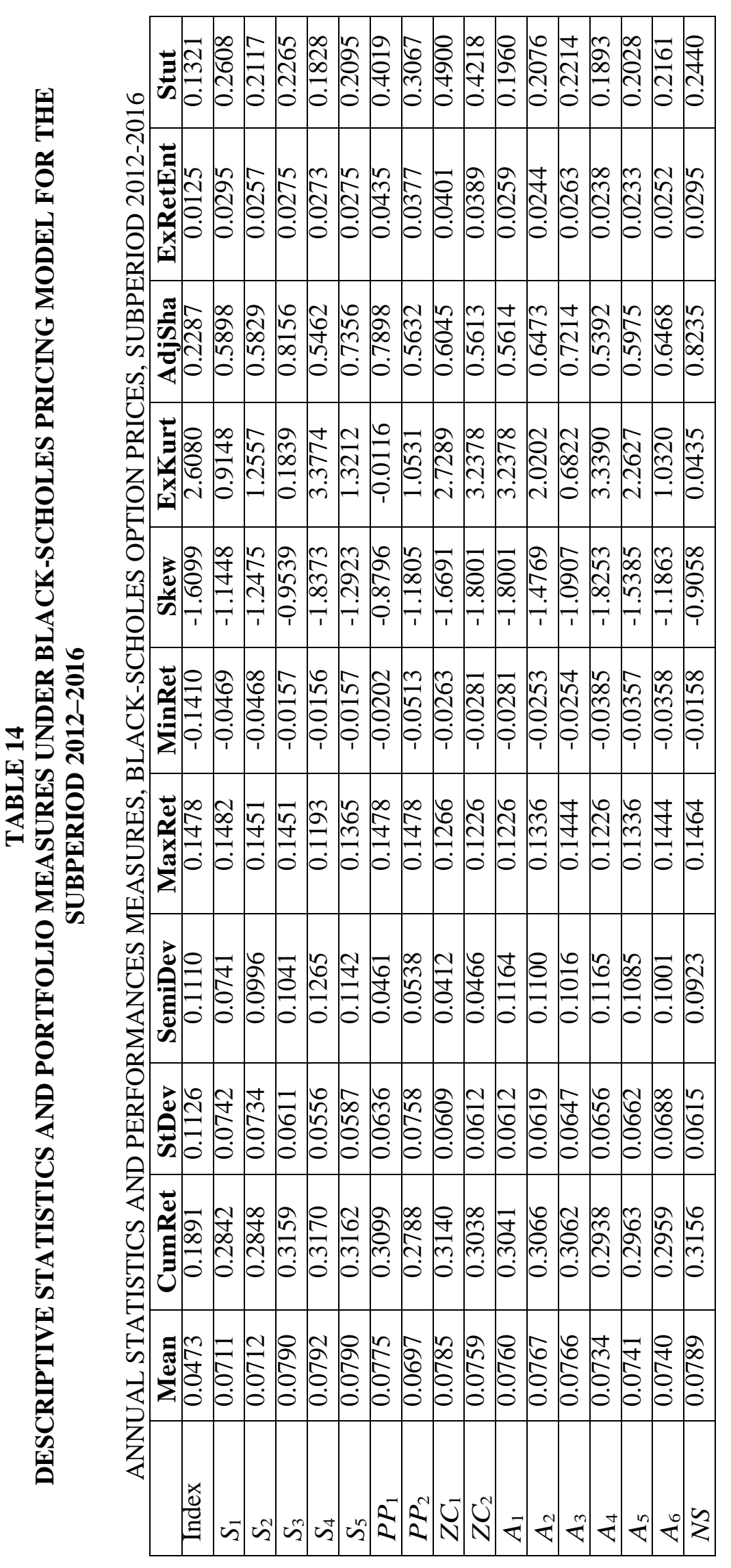

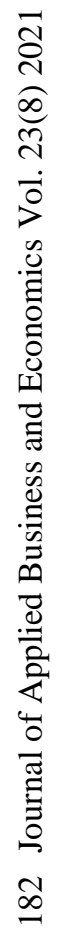


Percentage of Call Exercise Cost to the Initial Investment

The percentage in the table below is calculated as the total call exercise cost during the holding period, divided by the initial investment. It can be viewed as a risk measure of call exercise in the collar strategies.

FIGURE 10

PERCENTAGE OF CALL EXERCISE COST TO THE INITIAL INVESTMENT FOR ALL COLLAR PORTFOLIOS

\begin{tabular}{|c|c|c|c|c|c|c|c|c|}
\hline \multirow{2}{*}{ \% Call Exersize Cost } & \multicolumn{3}{|c|}{ Option Market Data Model } & \multicolumn{4}{c|}{ Black-Scholes Pricing Model } \\
\cline { 2 - 9 } & $\mathbf{2 0 0 8 - 2 0 1 6}$ & $\mathbf{2 0 0 8 - 2 0 1 2}$ & $\mathbf{2 0 1 0 - 2 0 1 4}$ & $\mathbf{2 0 1 2 - 2 0 1 6}$ & $\mathbf{2 0 0 8 - 2 0 1 6}$ & $\mathbf{2 0 0 8 - 2 0 1 2}$ & $\mathbf{2 0 1 0 - 2 0 1 4}$ & $\mathbf{2 0 1 2 - 2 0 1 6}$ \\
\hline S1 & $26 \%$ & $14 \%$ & $5 \%$ & $12 \%$ & $35 \%$ & $17 \%$ & $5 \%$ & $14 \%$ \\
\hline S2 & $37 \%$ & $16 \%$ & $10 \%$ & $22 \%$ & $49 \%$ & $21 \%$ & $11 \%$ & $24 \%$ \\
\hline S3 & $37 \%$ & $16 \%$ & $10 \%$ & $22 \%$ & $54 \%$ & $22 \%$ & $12 \%$ & $24 \%$ \\
\hline S4 & $64 \%$ & $24 \%$ & $24 \%$ & $40 \%$ & $93 \%$ & $38 \%$ & $27 \%$ & $41 \%$ \\
\hline S5 & $39 \%$ & $17 \%$ & $15 \%$ & $23 \%$ & $65 \%$ & $25 \%$ & $16 \%$ & $29 \%$ \\
\hline ZC1 & $39 \%$ & $21 \%$ & $10 \%$ & $12 \%$ & $24 \%$ & $17 \%$ & $5 \%$ & $5 \%$ \\
\hline ZC2 & $45 \%$ & $20 \%$ & $8 \%$ & $8 \%$ & $17 \%$ & $13 \%$ & $3 \%$ & $3 \%$ \\
\hline A1 & $57 \%$ & $24 \%$ & $23 \%$ & $31 \%$ & $86 \%$ & $38 \%$ & $27 \%$ & $35 \%$ \\
\hline A2 & $50 \%$ & $21 \%$ & $17 \%$ & $25 \%$ & $70 \%$ & $30 \%$ & $20 \%$ & $29 \%$ \\
\hline A3 & $38 \%$ & $16 \%$ & $9 \%$ & $18 \%$ & $59 \%$ & $25 \%$ & $15 \%$ & $24 \%$ \\
\hline A4 & $58 \%$ & $24 \%$ & $23 \%$ & $31 \%$ & $83 \%$ & $37 \%$ & $26 \%$ & $35 \%$ \\
\hline A5 & $51 \%$ & $18 \%$ & $14 \%$ & $20 \%$ & $68 \%$ & $29 \%$ & $20 \%$ & $29 \%$ \\
\hline A6 & $38 \%$ & $16 \%$ & $9 \%$ & $18 \%$ & $57 \%$ & $24 \%$ & $15 \%$ & $24 \%$ \\
\hline NS & $20 \%$ & $4 \%$ & $8 \%$ & $18 \%$ & $32 \%$ & $5 \%$ & $11 \%$ & $20 \%$ \\
\hline
\end{tabular}

Graphs of Comparison of Performance Measures for all Subperiods

The results are ordered with respect to Stutzer index. We normalized some values for visualization by multiplying the entire measure (except Stutzer index) by a constant.

FIGURE 11

COMPARISON OF PERFORMANCE MEASURE APPLIED TO PORTFOLIOS USING OMD MODEL FOR THE 2008-2012

\section{Option Market Data Model, subperiod 2008-2012}

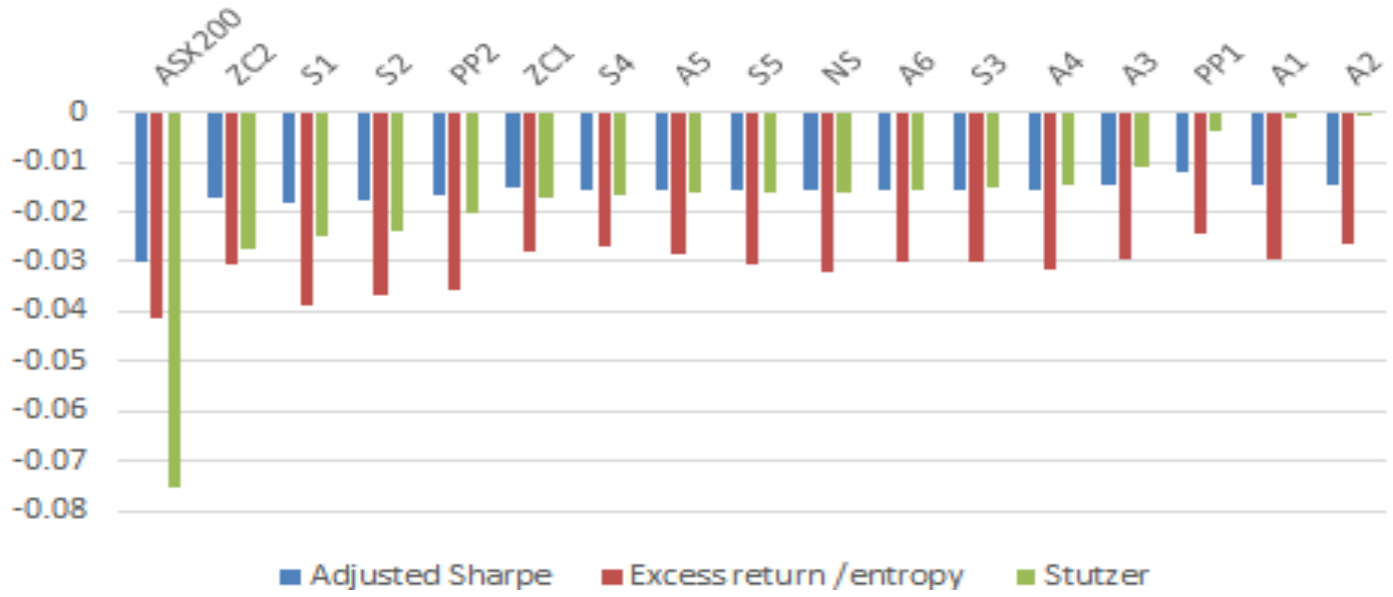


FIGURE 12

COMPARISON OF PERFORMANCE MEASURE APPLIED TO PORTFOLIOS USING BSP MODEL FOR THE SUBPERIOD 2008-2012

\section{Black-Scholes Option Pricing model, subperiod \\ 2008-2012}

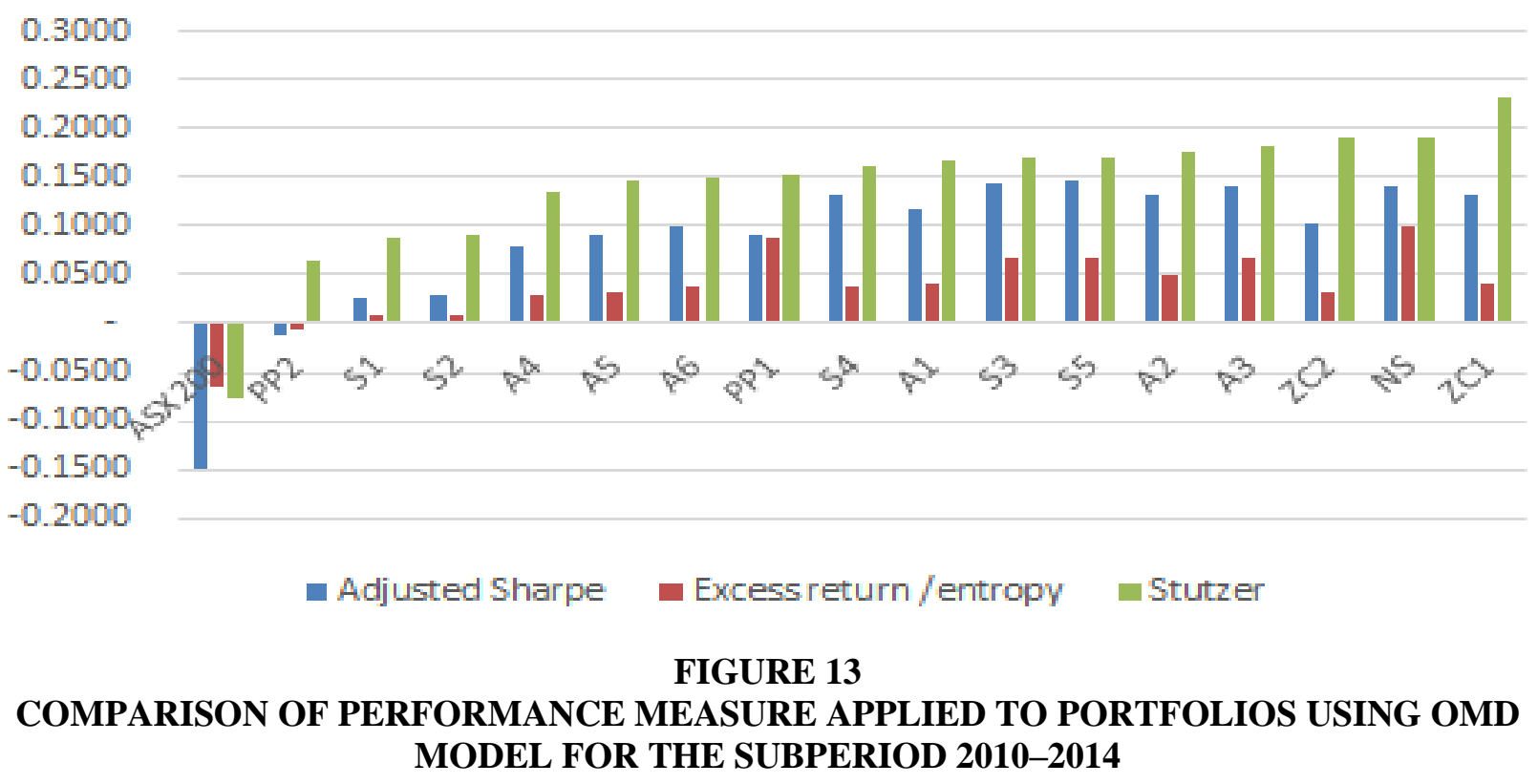

Option Market Data Model, subperiod 2010-2014

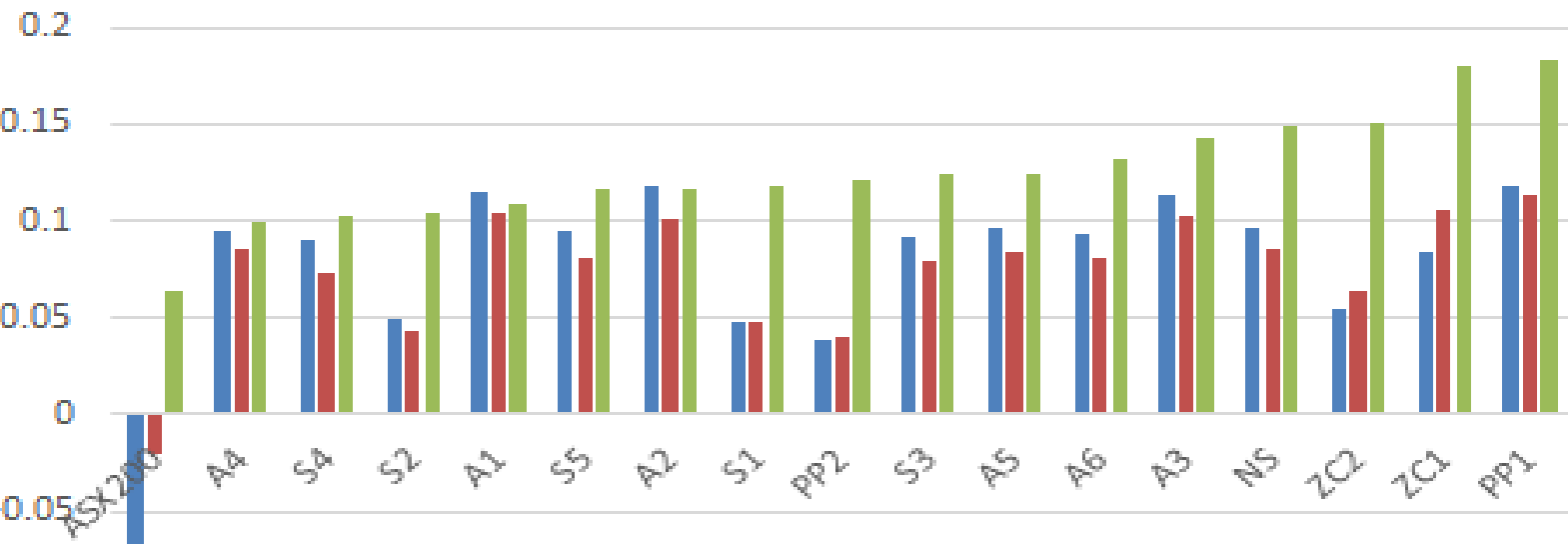

$-0.1$

adjusted Sharpe axcess return/entropy a Stutzer 
FIGURE 14

COMPARISON OF PERFORMANCE MEASURE APPLIED TO PORTFOLIOS USING BSP MODEL FOR THE SUBPERIOD 2010-2014

\section{Black-Scholes Option Pricing model, subperiod}

$$
\text { 2010-2014 }
$$

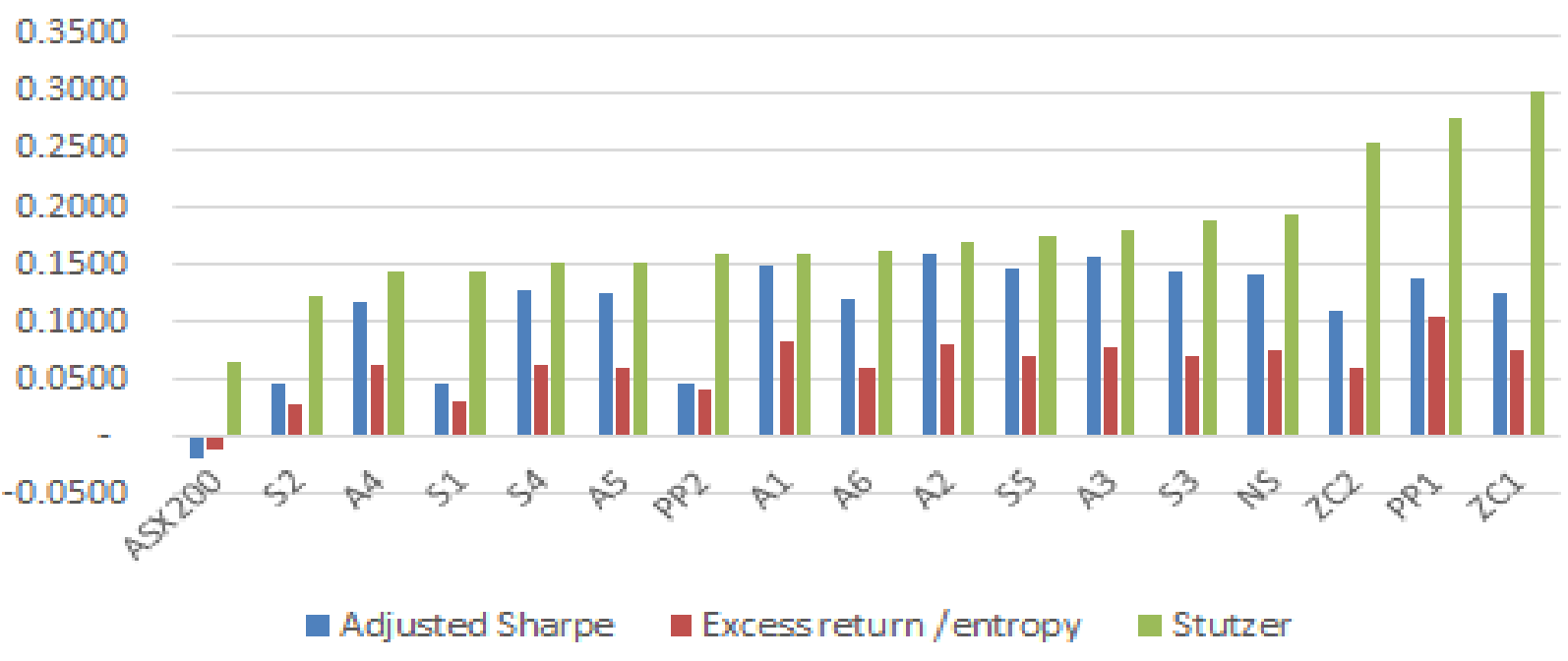

FIGURE 15

COMPARISON OF PERFORMANCE MEASURE APPLIED TO PORTFOLIOS USING OMD MODEL FOR THE SUBPERIOD 2012-2016

Option Market Data Model, subperiod 2012-2016

\subsection{5}

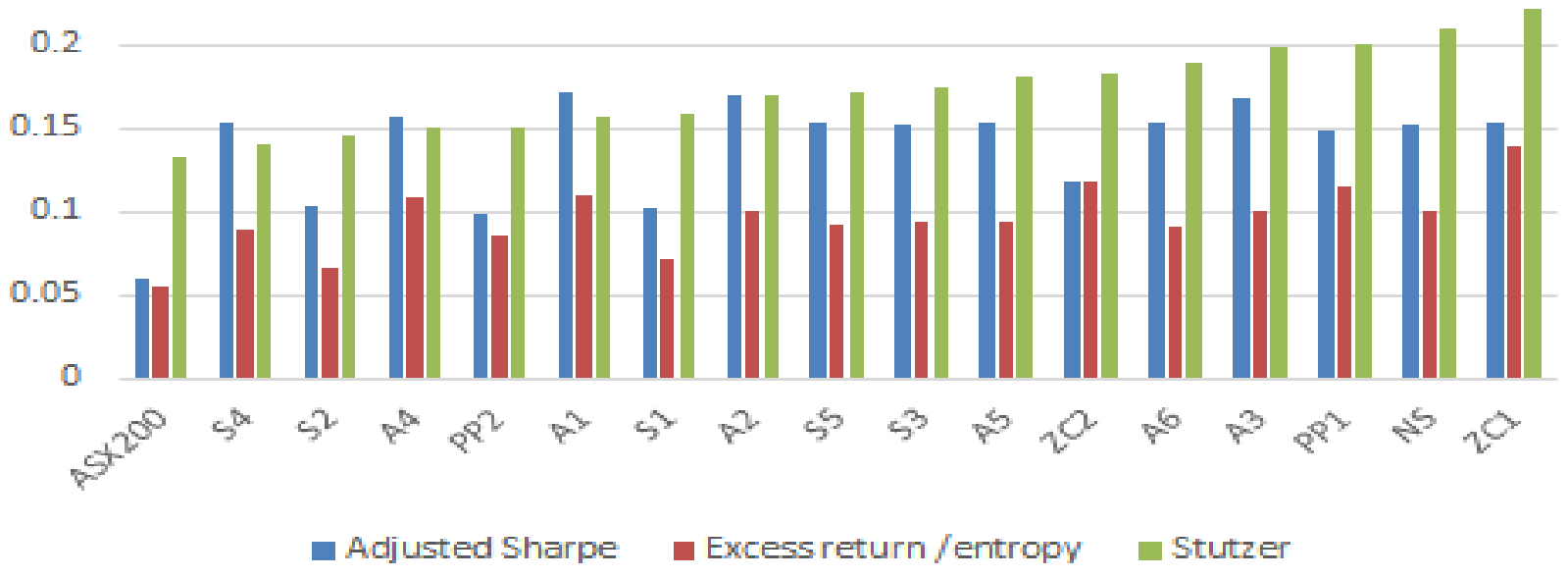


FIGURE 16

COMPARISON OF PERFORMANCE MEASURE APPLIED TO PORTFOLIOS USING BSP MODEL FOR THE SUBPERIOD 2010-2014

\section{Black-Scholes Option Pricing model, subperiod}

2012-2016

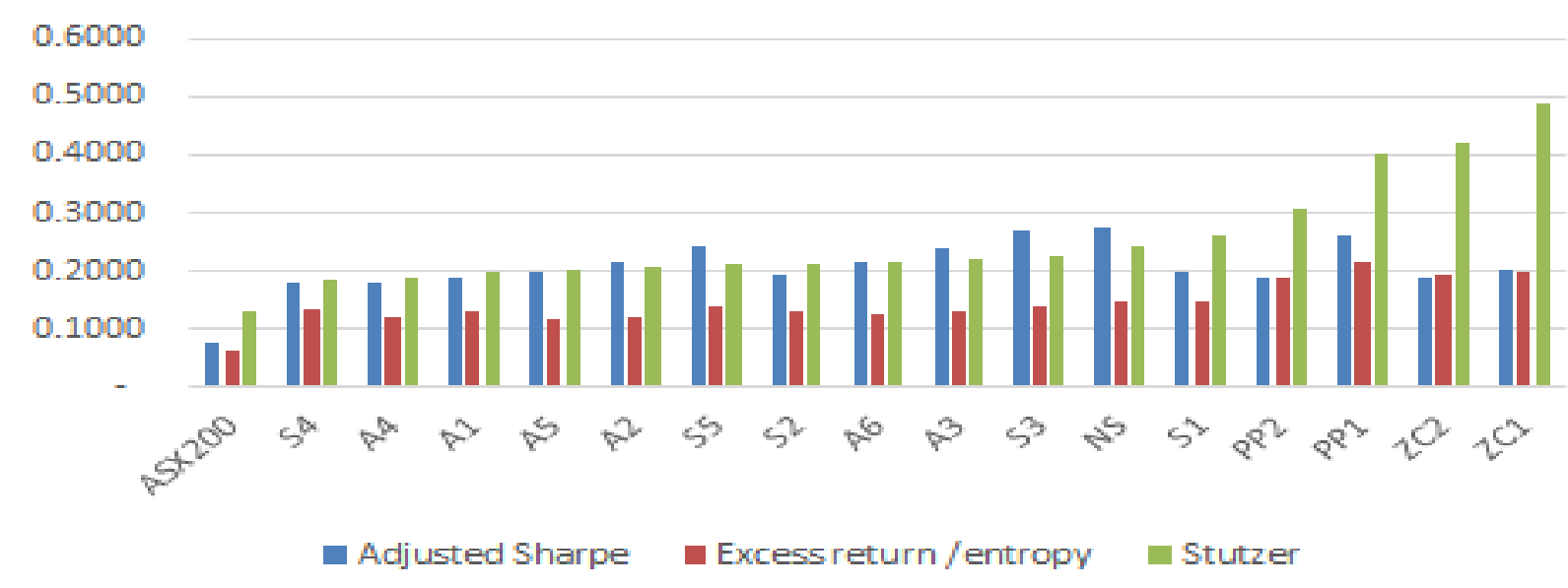

Rebalancing Dates

FIGURE 17

QUARTERLY REBALANCING DATES

\begin{tabular}{|c|c|c|c|c|c|}
\hline 20-Mar-08 & 17-Sep-09 & 17-Mar-11 & 20-Sep-12 & 20-Mar-14 & 17-Sep-15 \\
\hline 19-Jun-08 & 17-Dec-09 & 16-Jun-11 & 20-Dec-12 & 19-Jun-14 & 17-Dec-15 \\
\hline 18-Sep-08 & 18-Mar-10 & 15-Sep-11 & 21-Mar-13 & 18-Sep-14 & 17-Mar-16 \\
\hline 18-Dec-08 & 17-Jun-10 & 15-Dec-11 & 20-Jun-13 & 18-Dec-14 & 16-Jun-16 \\
\hline 19-Mar-09 & 16-Sep-10 & 15-Mar-12 & 19-Sep-13 & 19-Mar-15 & 15-Sep-16 \\
\hline 18-Jun-09 & 16-Dec-10 & 21-Jun-12 & 19-Dec-13 & 18-Jun-15 & \\
\hline
\end{tabular}

Formulae of Excess Return-Entropy Ratio, Adjusted Sharpe Ratio and Stutzer Index

Assume $X=\left\{x_{1}, x_{2}, \ldots, x_{m}\right\}$ and $Y=\left\{y_{1}, y_{2}, \ldots, y_{m}\right\}$ are two discrete random variables with probabilities $P=\left\{p_{i}=\mathbb{P}\left(X=x_{i}\right)\right\}$ and $Q=\left\{q_{i}=\mathbb{P}\left(Y=y_{i}\right)\right\}$. Then Shannon entropy, or simply entropy is defined by

$$
\mathcal{H}(P):=-\sum_{i=1}^{m} p_{i} \log p_{i}
$$

The Kullback-Leibler divergence (also called relative entropy) is given by

$$
D_{K L}(P \| Q):=-\sum_{i=1}^{m} p_{i} \log \frac{p_{i}}{q_{i}}
$$

We also use the convention that $0 \log 0=0$ which is easily justified by continuity since $x \log x \rightarrow 0$ as $x \rightarrow$ 0 . The Shannon entropy is positive, as $p_{i} \in[0,1]$ implies that $\log \frac{1}{p_{i}} \geq 0$. The excess return ratio is simply the excess return divided by the Shannan entropy (see Philippatos \& Wilson (1972)). 
Denoting the Sharpe ratio (excess return divided by the standard deviation) by S, the Adjusted Sharpe ratio $A S R$ is given by

$$
A S R=S+\frac{\rho}{6} S^{2}-\frac{\mathrm{K}}{24} S^{3}
$$

where $\rho$ is the skewness and $\mathrm{k}$ is the kurtosis (p. 49 of Bacon (2013)).

The performance index introduced by Stutzer (2000) is based on maximizing the rate at which the probability of performance being smaller than zero approaches to zero as time goes to infinity. That is called Stutzer's information statistic, given by

$$
I_{P}=\max _{\theta}\left(-\log \left(\frac{1}{T} \sum_{i=1}^{T} \exp \left(\theta r_{t}\right)\right)\right)
$$

where $r_{t}$ is the excess return of the portfolio. When the returns are normally distributed, Stutzer's information statistic, $I_{P}$ is equal to half of the squared Sharpe ratio, $\lambda_{P}$. That is,

$$
I_{P}=\frac{1}{2} \lambda_{P}^{2} \quad \Leftrightarrow \quad \sqrt{2 I_{P}}=\lambda_{P}
$$

The Stutzer index, Stut is formulated as

$$
\text { Stut }:=\frac{|\mu|}{\mu} \sqrt{2 I_{P}}
$$

where $\mu$ is the mean excess return. 